\title{
ON SOME CLASSES OF CURVES IN A PROJECTIVE SPACE
}

\author{
VYACHESLAV SEDYKH \\ Department of Mathematics \\ Gubkin University of Oil and Gas \\ Leninsky prosp. 65, Moscow 117917, Russia \\ E-mail:sedykh@ium.ips.ras.ru
}

1. The main result. $A$ closed curve in the $n$-dimensional real projective space $\mathbb{R} P^{n}$ is any $C^{\infty}$-mapping of a circle into this space. A curve is called smooth if it is embedded.

For every point of a curve in $\mathbb{R} P^{n}$, there is a hyperplane intersecting the curve at this point at least $n$ times. Such a hyperplane is called osculating hyperplane to the curve at a given point. A point of a curve is said to be its flattening point if the multiplicity of the intersection of the curve with an osculating hyperplane at this point is greater than $n$.

Definition 1. A curve in $\mathbb{R} P^{n}$ is called convex if any hyperplane intersects it at no more than $n$ points, taking the multiplicities into account.

A convex curve has no flattening points. Such a curve in an even-dimensional projective space is contractible and affine, it is not contractible in an odd-dimensional space $([\mathrm{An}])$. A smooth closed curve in $\mathbb{R} P^{n+1}$ which is projected into a convex curve in $\mathbb{R} P^{n}$ from a point lying outside of the curve has at least $n+2$ geometrically different flattening points $([\mathrm{Ar}])$.

Definition 2. A curve in $\mathbb{R} P^{n+1}$ is said to be convex by Barner if for any of its $n$ points (not necessarily geometrically different) there is a hyperplane which passes through these points and does not intersect the curve anymore.

A closed convex by Barner curve in an odd-dimensional projective space is contractible and affine. Such a curve in an even-dimensional space is not contractible. A smooth closed convex by Barner curve in $\mathbb{R} P^{n+1}$ has at least $n+2$ geometrically different flattening points $([\mathrm{B}])$.

1991 Mathematics Subject Classification: Primary 51L15; Secondary 52A20.

Partially supported by RFBR and INTAS.

The paper is in final form and no version of it will be published elsewhere. 
Let us equip the space of all closed curves in a projective space by the $C^{\infty}$-topology. Convex curves and convex by Barner curves form open subsets in this space.

A curve in $\mathbb{R} P^{n+1}$ projected into a convex curve in $\mathbb{R} P^{n}$ from a point lying outside of the curve is convex by Barner. We have the natural question (V. I. Arnold, 1996): what is the relation between these two classes of curves? The answer is given by the following

THEOREM 1. There is an open set of smooth closed curves in $\mathbb{R} P^{n+1}$ which are convex by Barner and have no convex projections into any hyperplane.

The proof of this statement is given in Section 5. It is based on some general facts on convex curves (Section 2) and properties of some special families of curves (Sections 3 and 4 ). The proof of these properties is given in the second part of the paper (Sections 6 and 7$)$.

2. General facts on convex curves. A flattening point of a curve in $\mathbb{R} P^{n}$ is called $k$-multiple if the multiplicity of the intersection of the curve with its osculating hyperplanes at this point does not exceed $n+k$ and is equal to $n+k$ with one of them. Flattening points of the multiplicity $k>1(k=1)$ are called multiple (nonmultiple, respectively).

Remark 1. If a curve belongs to the boundary of the set of convex curves in the space of all closed curves in $\mathbb{R} P^{n}$, then it has no nonmultiple flattening points. Indeed, if $M$ is such a point, then there is a hyperplane which transversally intersects the curve at $n+1$ geometrically different points being close to $M$. This follows from properties of the versal deformation $F(x, \lambda)=x^{n+1}+\lambda_{1} x^{n}+\ldots+\lambda_{n} x+\lambda_{n+1}$ of the function $x^{n+1}$ (the singularity $A_{n}$; see $[\mathrm{AGV}]$ ). Namely, for any $i=1, \ldots, n+1$ there is $\lambda=$ $\left(\lambda_{1}, \ldots, \lambda_{i-1}, 0, \lambda_{i+1}, \ldots, \lambda_{n+1}\right)$ arbitrarily close to 0 and such that the function $F(x, \lambda)$ has $n+1$ nonmultiple real zeros (the last is a simple exercise on symmetric functions).

An osculating hyperplane at every nonflattening point of a curve is unique. Flattening points where an osculating hyperplane is not unique are called inflection points.

Remark 2. Let a closed curve in $\mathbb{R} P^{n}$ belong to the boundary of the set of convex curves and have no inflection points. Then:

1) this curve has no flattening points of any odd multiplicity (otherwise, any close curve would have flattening points);

2) any hyperplane which intersects the curve more than $n$ times (taking the multiplicities into account) has only multiple intersection points with it (otherwise, any close curve would intersect some hyperplane at least at $n+1$ points).

Re mark 3 . If a closed curve $\gamma$ in $\mathbb{R} P^{n}$ belongs to the boundary of the set of convex curves, has no inflection points and the multiplicity of its flattening points is not greater than 2 , then the osculating hyperplane at every of its points does not intersect the curve at other points. Indeed, if the osculating hyperplane $\pi$ to $\gamma$ at a point $M$ intersects $\gamma$ at some point $M^{\prime} \neq M$, then there is a hyperplane close to $\pi$, passing through $M^{\prime}$ and transversally intersecting $\gamma$ at $n$ geometrically different points being close to $M$ (as above, the existence of $n$ such points follows from properties of the versal deformation of the singularity $A_{\mu}$ where $\mu$ is equal to $n-1$ and $\left.n+1\right)$. 
DEFINITION 3. We say that a curve satisfies the generic condition ( $\star$ ) if an osculating hyperplane at every of its points is unique and does not intersect the curve at other points.

A curve satisfying the generic condition $(\star)$ is smooth.

THEOREM 2. Let a curve $\gamma$ belong to the boundary of the set of convex curves in the space of all closed curves in $\mathbb{R} P^{n}$. Suppose that $\gamma$ satisfies the generic condition $(\star)$. Then any hyperplane $\pi$ which intersects $\gamma$ more than $n$ times (taking the multiplicities into account) is the osculating hyperplane to $\gamma$ at a multiple flattening point.

Remark 4. The fact that the set $\gamma \cap \pi$ contains a multiple flattening point of the curve $\gamma$ is proved in [An] without any conditions of general position. The author considers a central projection of $\gamma$ and applies the induction on $n$. But the validity of the inductive step is not proved. The point is that a central projection of the curve $\gamma$ can be nonsmooth (for example, $\gamma$ can have pieces lying in a hyperplane). To prove the validity of the inductive step, we use the additional generic condition $(\star)$.

Proof. For $n=1$ the statement is obvious. Suppose that it is true for a space of the dimension $n-1$ and prove it in $\mathbb{R} P^{n}$.

Let $M$ be an intersection point of the curve $\gamma$ with the hyperplane $\pi$. It is a multiple intersection point according to Remark 2. Consider projective lines intersecting $\gamma$ at least at two points (taking the multiplicities into account) one of which is $M$. By condition ( $\star$ ), these lines define a smooth closed curve $\gamma_{M}$ in $\mathbb{R} P^{n-1}$.

The curve $\gamma_{M}$ belongs to the boundary of the set of convex curves in the space of all closed curves in $\mathbb{R} P^{n-1}([\mathrm{An}])$. Projective lines lying in the hyperplane $\pi$ and passing through $M$ form the hyperplane $\pi^{\prime}$ in $\mathbb{R} P^{n-1}$ which intersects $\gamma_{M}$ more than $n-1$ times (taking the multiplicities into account). One of points of this intersection is the tangent line $M^{\prime}$ to the curve $\gamma$ at the point $M$.

It is easy to see that the curve $\gamma_{M}$ satisfies the condition $(\star$ ) (as in Remark 1, it is needed to consider the versal deformation of the singularity $\left.A_{n-2}\right)$. Hence by the induction hypothesis, $\pi^{\prime}$ is the osculating hyperplane to $\gamma_{M}$ at the point $M^{\prime}$ which is a multiple flattening point of the curve. This means that $\pi$ is the osculating hyperplane to $\gamma$ at the multiple flattening point $M$.

COROllary 1 (see $[\mathrm{Ar}])$. A closed curve in a projective space belonging to the boundary of the set of convex curves has flattening points. All of them are multiple.

Proof. A closed curve without flattening points cannot belong to the boundary of the set of convex curves. Otherwise, it would satisfy the generic condition $(\star)$ (Remark 3 ) and hence, would have multiple flattening points (Theorem 2). Thus, any closed curve belonging to the boundary of the set of convex curves has flattening points. Their multiplicity is greater than 1 (Remark 1$)$.

THEOREM 3. Let $\Gamma_{t}, t \in[0,1]$, be a continuous family of smooth closed curves in $\mathbb{R} P^{n+1}$ and $M \in \mathbb{R} P^{n+1} \backslash \bigcup_{t} \Gamma_{t}$. Suppose that the projection $\gamma_{t} \subset \mathbb{R} P^{n}$ of the curve $\Gamma_{t}$ from the point $M$ is convex for any $t \in[0,1)$ and is not convex for $t=1$. Let the curves $\Gamma_{1}$ and $\gamma_{1}$ satisfy the following conditions: 
(A) an osculating hyperplane to $\Gamma_{1}$ at every point is unique;

(B) connected components of the set of flattening points of the curve $\Gamma_{1}$ containing more than one point are convex by Barner;

(C) every hyperplane in $\mathbb{R} P^{n}$ intersecting the curve $\gamma_{1}$ more than $n$ times (taking the multiplicities into account) intersects it by a connected subset on a circle consisting of multiple flattening points.

Then the curve $\Gamma_{1}$ is convex by Barner.

Proof. By condition (C), any hyperplane in $\mathbb{R} P^{n+1}$ passing through the point $M$ and $n$ points of the curve $\Gamma=\Gamma_{1}$ which are not necessarily mutually different but such that all of them are not projected simultaneously into one of the connected components of the set of flattening points of the curve $\gamma=\gamma_{1}$ have no other common points with $\Gamma$.

Now, let us consider a hyperplane $\pi$ in $\mathbb{R} P^{n+1}$ which passes through the point $M$ and is projected from this point into a hyperplane $\pi^{\prime}$ in $\mathbb{R} P^{n}$ intersecting the curve $\gamma$ with the multiplicity greater than $n$. The hyperplane $\pi$ intersects the curve $\Gamma$ by the connected set $K$ since this intersection is projected into the connected (in the standard topology of a circle) set $\pi^{\prime} \cap \gamma$ (condition (C)). Moreover, since the intersection $\pi^{\prime} \cap \gamma$ consists of multiple flattening points of the curve $\gamma$, then $K$ is a connected component of the set of flattening points of the curve $\Gamma$ and $\pi$ is an osculating hyperplane to this curve at any point of $K$.

Let us show that for any set of points $M_{1}, \ldots, M_{l}$ from $K$ and any set of natural numbers $k_{1}, \ldots, k_{l}$ such that $k_{1}+\ldots+k_{l}=n$, one can turn the hyperplane $\pi$ so that the new hyperplane will intersect the curve $\Gamma$ only at points $M_{1}, \ldots, M_{l}$ and with multiplicities $k_{1}, \ldots, k_{l}$, respectively. Indeed, let us choose homogeneous coordinates $\left(x_{0}: x_{1}: \ldots: x_{n+1}\right)$ in $\mathbb{R} P^{n+1}$ so that:

1) the curve $\Gamma$ is defined by the formulas $x_{j}=x_{j}(s), j=0,1, \ldots, n+1$, where $x_{0}(s), \ldots, x_{n+1}(s)$ are $C^{\infty}$-functions on a circle and

$$
\begin{cases}x_{n+1}(s)=x_{n+1}^{\prime}(s)=\ldots=x_{n+1}^{(n+1)}(s)=0, & \text { if } s \in K, \\ x_{n+1}(s) \neq 0, & \text { if } s \notin K ;\end{cases}
$$

2) the hyperplane $\pi$ is defined by the equation $x_{n+1}=0$;

3) the hyperplane $x_{n}=0$ intersects $\Gamma$ at points $M_{1}, \ldots, M_{l}$ with the multiplicities not less than $k_{1}, \ldots, k_{l}$, respectively.

It is easy to see that the function $x_{n+1}(s)$ changes its sign when $s$ passes through $K$, if $n$ is odd, and does not change the sign, if $n$ is even (this follows from the fact that the curve $\Gamma$ as a continuous limit of smooth closed convex by Barner curves is not contractible for odd $n$ and contractible for even $n)$. The function $x_{n}(s)$ has exactly $n$ zeros $M_{1}, \ldots, M_{l}$ with the multiplicities $k_{1}, \ldots, k_{l}$, respectively, in some neighbourhood $U$ of the set $K$ (this follows from condition (A), if $K$ consists of one point, and from condition (B), if $K$ contains more than one point). Thus, we can define a smooth function

$$
y(s)= \begin{cases}0, & \text { if } s \in K, \\ x_{n+1}(s) / x_{n}(s), & \text { if } s \in U \backslash K,\end{cases}
$$

in the neighbourhood $U$ which does not change its sign when $s$ passes through $K$. 
Let us fix an arbitrary $\varepsilon \neq 0$ such that $y(s)+\varepsilon \neq 0$ for every $s \in U$, and consider the hyperplane $\pi_{\varepsilon}$ given by the equation $x_{n+1}+\varepsilon x_{n}=0$. The intersection points of this hyperplane with the curve $\Gamma$ in the neighbourhood $U$ are defined by the equation $x_{n}(s)[y(s)+\varepsilon]=0$. Hence, the hyperplane $\pi_{\varepsilon}$ intersects the curve $\Gamma$ at the points $M_{1}, \ldots, M_{l}$, with the multiplicities $k_{1}, \ldots, k_{l}$, respectively, and does not intersect it at other points of the neighbourhood $U$. But for sufficiently small $\varepsilon$, this hyperplane does not intersect $\Gamma$ at all other points as well since the curve is closed.

Re mark 5. Generic finitely-parametric families of curves in a projective space satisfy condition (B).

Remark 6. Theorem 1 does not follow directly from Theorem 3. Indeed, it is easy to construct a family of curves $\Gamma_{t}$ with indicated properties whose projections from the point $M$ lose the convexity at $t=1$. But the curve $\Gamma_{1}$ can have convex projections from other points of the ambient space.

TheOREM 4. Let $\gamma$ be a smooth closed curve in $\mathbb{R} P^{n+1}$. Then the set $\Omega$ of points in $\mathbb{R} P^{n+1} \backslash \gamma$ such that $\gamma$ is projected from them into a convex curve is either empty or a connected component of the complement in $\mathbb{R} P^{n+1}$ to the union $\Delta$ of the osculating hyperplanes at flattening points of the curve $\gamma$.

P r o of. The set $\Omega$ is open since the set of convex curves in $\mathbb{R} P^{n}$ is open. In addition, $\Omega \subset \mathbb{R} P^{n+1} \backslash \Delta$ because the projection of the curve $\gamma$ from a point in $\mathbb{R} P^{n+1} \backslash \gamma$ belonging to an osculating hyperplane to $\gamma$ intersects the projection of this hyperplane more than $n$ times (taking the multiplicities into account).

Suppose that $\Omega \neq \emptyset$ (in particular, the curve $\gamma$ has unique osculating hyperplane at every point). Let $M$ be a boundary point of the set $\Omega$ which is not a flattening point of the curve $\gamma$. Then $M \notin \gamma$. Indeed, if $M \in \gamma$, then through any point $M^{\prime} \in \Omega$ sufficiently close to $M$, one can pass a hyperplane intersecting the curve $\gamma$ at $n+1$ points. But this contradicts the fact that the projection of the curve $\gamma$ from the point $M^{\prime}$ is convex.

Hence, the projection $\gamma_{M}$ of the curve $\gamma$ from the point $M$ belongs to the boundary of the set of convex curves in the space of all closed curves in $\mathbb{R} P^{n}$. According to Corollary 1 the curve $\gamma_{M}$ has multiple flattening points. Thus, $M \in \Delta$ and $\Omega$ is the union of connected components of the set $\mathbb{R} P^{n+1} \backslash \Delta$.

Let us prove that the set $\Omega$ is connected. For this, consider the cases of even and odd $n$ separately.

1) $n$ is even. In this case the space $\mathbb{R} P^{n+1}$ is oriented and the curve $\gamma$ is affine since it has convex projections from points lying outside of the curve. Let us orient $\mathbb{R} P^{n+1}$ and fix a parametrization of the curve $\gamma$.

Let $M \in \mathbb{R} P^{n+1} \backslash \gamma$. Then the cone with the vertex $M$ and the directrix $\gamma$ is divided by the point $M$ and the curve $\gamma$ onto two parts. Hence, there exists a smooth family of nonzero vectors $v(s) \in T_{s} \mathbb{R} P^{n+1}, s \in \gamma$, which are tangent to projective lines passing through the points $M$ and $s$.

Consider the set $\mathcal{E}(s)$ of vectors in $T_{s} \mathbb{R} P^{n+1}, s \in \gamma$, consisting of the leading $n$ derivatives of the curve $\gamma$ at the point $s$ and the vector $v(s)$. If $M \in \Omega$, then the set $\mathcal{E}(s)$ is linearly independent for any $s$ and all these sets are oriented identically. 
If the point $M$ passes through the osculating hyperplane to the curve $\gamma$ at a flattening point $s$, then the orientation of the set $\mathcal{E}(s)$ is changed to the opposite one. Hence, for the replacement of the point $M$ into the set $\Omega$ when it moves along any projective line, it is necessary that it crosses the osculating hyperplanes to $\gamma$ at all flattening points. Then obviously, it goes back to the same connected component of the set $\mathbb{R} P^{n+1} \backslash \Delta$.

2) $n$ is odd. Let $\pi$ be the osculating hyperplane to the curve $\gamma$ at one of its flattening points (such a point necessarily exists since $\gamma$ has convex projections $[\mathrm{Ar}]$ ). Let us orient the hyperplane $\pi \cong \mathbb{R} P^{n}$ and fix a parametrization of $\gamma$.

The orientations of the moving $n$-hedrons for the projection $\gamma_{M}$ of the curve $\gamma$ into the hyperplane $\pi$ from any point $M \in \Omega$ are the same at all points of the curve $\gamma_{M}$. If the point $M$ passes through the osculating hyperplane to the curve $\gamma$ at a flattening point $s$, then the orientation of the moving $n$-hedron for the curve $\gamma_{M}$ at the point being the image of a point $s$ under the projection from the point $M$ is changed to the opposite one. Hence, as above, for the replacement of the point $M$ into the set $\Omega$ when it moves along any projective line, it is necessary that it crosses the osculating hyperplanes to $\gamma$ at all flattening points and thus finds oneself into the same connected component of the set $\mathbb{R} P^{n+1} \backslash \Delta$.

3. The family of curves in $\mathbb{R} P^{n+1}, n=2 m$. Let $0 \leq t<1$ and $r(\varphi)=1-$ $t \sin ^{2}(m+1) \varphi$. Let us take homogeneous coordinates $\left(x_{0}: \ldots: x_{2 m+1}\right)$ in $\mathbb{R} P^{2 m+1}$ and consider the family of smooth closed curves $\Gamma_{t}: S^{1} \rightarrow \mathbb{R} P^{2 m+1}$,

$$
\begin{gathered}
x_{0}=1, \quad x_{1}=r(\varphi) \cos \varphi, \quad x_{2}=r(\varphi) \sin \varphi, \\
x_{3}=\cos 2 \varphi, \quad x_{4}=\sin 2 \varphi, \quad \ldots, \quad x_{2 m-1}=\cos m \varphi, \quad x_{2 m}=\sin m \varphi, \\
x_{2 m+1}=f(\varphi)=\sin \left[(m+1) \varphi+\left(1+(-1)^{m}\right) \frac{\pi}{4}\right], \quad\{\varphi \bmod 2 \pi\},
\end{gathered}
$$

depending on the parameter $t$. The projection $\gamma_{t}$ of the curve $\Gamma_{t}$ from the point $\Theta=$ $(0: \ldots: 0: 1)$ into the hyperplane $\Pi$ given by the equation $x_{2 m+1}=0$ is a smooth closed curve in $\mathbb{R} P^{2 m}$. For $t=0$, the curve $\gamma_{t}$ is convex ([Sch]).

Proposition 1. For any $t \in\left[0, t_{0}\right)$ where

$$
\frac{1}{t_{0}}=\frac{1}{2}+\frac{(3 m+1) !\left(10 m^{3}+24 m^{2}+15 m+2\right)}{4 m !((m+2) !)^{2}} \geq 9,
$$

the curve $\gamma_{t}$ has no flattening points. For $t=t_{0}$, the curve $\gamma_{t}$ has exactly $2 m+2$ flattening points (2-multiple) given by the equation

$$
\cos 2(m+1) \varphi=(-1)^{m} .
$$

For any $t>t_{0}$ sufficiently close to $t_{0}$, the curve $\gamma_{t}$ has exactly $4 m+4$ flattening points.

The proof is given in Subsection 6.2.

Remark 7 . The sequence of numbers $t_{0}$ exponentially decreases $\left(\sim m / 27^{m}\right)$ when $m$ increases. The beginning of this sequence is:

$$
\frac{1}{9}, \quad \frac{1}{228}, \quad \frac{1}{5597}, \quad \frac{4}{543545}, \quad \frac{2}{6613465}, \quad \frac{4}{323831081}, \ldots .
$$


Proposition 2. For any $t \in\left[0, T_{0}\right)$ where

$$
\frac{1}{t_{0}}>\frac{1}{T_{0}}=\frac{1}{2}+\frac{(3 m+2) !(4 m+5)}{4 m !((m+2) !)^{2}} \geq 8,
$$

the curve $\Gamma_{t}$ has exactly $2 m+2$ (nonmultiple) flattening points given by equation (2). For $t=T_{0}$, the curve $\Gamma_{t}$ has exactly $4 m+4$ flattening points given by the equation $\sin 2(m+1) \varphi=0$.

The proof is given in Subsection 6.6.

Rem ark 8 . The sequence of numbers $T_{0}$ exponentially decreases $\left(\sim m^{2} / 27^{m}\right)$ when $m$ increases. The beginning of this sequence is:

$$
\frac{1}{8}, \quad \frac{4}{457}, \quad \frac{1}{1964}, \quad \frac{4}{147149}, \quad \frac{2}{1458601}, \quad \frac{4}{60276647}, \ldots .
$$

4. The family of curves in $\mathbb{R} P^{n+1}, n=2 m-1$. Let us take homogeneous coordinates $\left(x_{1}: \ldots: x_{2 m+1}\right)$ in $\mathbb{R} P^{2 m}$ and consider the family of smooth closed curves $\Gamma_{t}: S^{1} \rightarrow \mathbb{R} P^{2 m}$

$$
x_{1}=\cos \varphi-t \cos (4 m+3) \varphi, \quad x_{2}=\sin \varphi-t \sin (4 m+3) \varphi,
$$

(3) $x_{3}=\cos 3 \varphi, \quad x_{4}=\sin 3 \varphi, \quad \ldots, \quad x_{2 m-1}=\cos (2 m-1) \varphi, \quad x_{2 m}=\sin (2 m-1) \varphi$,

$$
x_{2 m+1}=f(\varphi)=\sin \left[(2 m+1) \varphi+\left(1+(-1)^{m}\right) \frac{\pi}{4}\right], \quad\{\varphi \bmod \pi\},
$$

depending on the parameter $t \in[0,1)$. The projection $\gamma_{t}$ of the curve $\Gamma_{t}$ from the point $\Theta=(0: \ldots: 0: 1)$ into the hyperplane $\Pi$ given by the equation $x_{2 m+1}=0$ is a smooth closed curve in $\mathbb{R} P^{2 m-1}$. For $t=0$, the curve $\gamma_{t}$ is convex $([\mathrm{Ar}])$.

Proposition 3. For any $t \in\left[0, t_{0}\right)$ where

$$
t_{0}=\frac{2(2 m+1)(m-1) !((m+1) !)^{2}}{(4 m+3)(3 m+1) !} \leq \frac{1}{7}
$$

the curve $\gamma_{t}$ has no flattening points. For $t=t_{0}$, the curve $\gamma_{t}$ has exactly $2 m+1$ flattening points (2-multiple) given by the equation

$$
\cos 2(2 m+1) \varphi=(-1)^{m-1} .
$$

For every $t>t_{0}$ sufficiently close to $t_{0}$, the curve $\gamma_{t}$ has exactly $4 m+2$ flattening points.

Re mark 9 . The sequence of numbers $t_{0}$ exponentially decreases $\left(\sim m / 27^{m}\right)$ when $m$ increases. The beginning of this sequence is:

$$
\frac{1}{7}, \quad \frac{1}{154}, \quad \frac{1}{3375}, \quad \frac{1}{76076}, \quad \frac{1}{1758120}, \quad \frac{1}{41442192}, \ldots .
$$

The proof is given in Subsection 7.2.

Proposition 4. For any $t \in\left[0, T_{0}\right)$ where

$$
t_{0}<T_{0}=\frac{2(2 m+1)(m+1)^{2}(m !)^{3}}{(3 m+2) !} \leq \frac{1}{5},
$$


the curve $\Gamma_{t}$ has exactly $2 m+1$ (nonmultiple) flattening points given by equation (4). For $t=T_{0}$, the curve $\Gamma_{t}$ has exactly $4 m+2$ flattening points given by the equation $\sin 2(2 m+1) \varphi=0$.

The proof is given in Subsection 7.6.

Remark 10. The sequence of numbers $T_{0}$ exponentially decreases $\left(\sim m^{2} / 27^{m}\right)$ when $m$ increases. The beginning of this sequence is:

$$
\frac{1}{5}, \quad \frac{1}{56}, \quad \frac{1}{825}, \quad \frac{1}{14014}, \quad \frac{1}{259896}, \quad \frac{1}{5116320}, \ldots .
$$

5. Proof of Theorem 1. Let $\Gamma_{t}$ be the family of curves in $\mathbb{R} P^{n+1}$ from Section 3 (or 4 ) and $\gamma_{t}$ be the projection of the curve $\Gamma_{t}$ from the point $\Theta$ into the hyperplane $\Pi$.

The curves $\gamma_{t}$ have no flattening points for $t \in\left[0, t_{0}\right)$ by Proposition 1 (or 3 ). Hence, they are convex by Corollary 1 and the convexity of the curve $\gamma_{0}$. In particular, the curve $\gamma_{t_{0}}$ belongs to the boundary of the set of convex curves in the space of all closed curves in $\mathbb{R} P^{n}$ since it has flattening points.

Proposition 5. The curves $\Gamma_{t_{0}}$ and $\gamma_{t_{0}}$ have unique osculating hyperplane at every point.

This is evident for points $\Gamma_{t_{0}}(\varphi)$ and $\gamma_{t_{0}}(\varphi)$ such that $\varphi$ is not a flattening point of the curve $\gamma_{t_{0}}$. The proof for other points is given in Subsections 6.4 and 7.4.

Remark 11. The curve $\gamma_{t_{0}}$ satisfies the generic condition $(\star)$ according to Propositions 1 (or 3), 5 and Remark 3. The curve $\Gamma_{t_{0}}$ satisfies the conditions of Theorem 3: (A) by Proposition 5; (B) by Proposition 2 (or 4); (C) by Theorem 2 .

Let us consider the union $\Delta_{t}$ of osculating hyperplanes to the curve $\Gamma_{t}$ at its flattening points. If $t$ is sufficiently close to $t_{0}$, then the set $\Delta_{t}$ is the union of $n+2$ osculating hyperplanes to the curve $\Gamma_{t}$ at points given by the equation (2) (or (4)). This follows from Propositions 2 (or 4) and 5.

The following properties of the set $\Delta_{t}$ are proved in Sections 6 and 7 .

Proposition 6. The intersection of any $n+1$ hyperplanes from $\Delta_{t_{0}}$ is equal to $\Theta$.

Proposition 7. For any $t \neq t_{0}$ sufficiently close to $t_{0}$, the intersection of all hyperplanes from $\Delta_{t}$ is empty.

Proposition 8. For any solution $\varphi$ of the equation (2) (or (4)), the family of osculating hyperplanes to the curves $\Gamma_{t}$ at the point $\varphi$ transversally intersects the point $\Theta$ (as a hyperplane in the dual space) at $t=t_{0}$.

According to Propositions 6 and 7 , the set $\mathbb{R} P^{n+1} \backslash \Delta_{t}$ has exactly $2^{n+1}$ connected components for every $t \neq t_{0}$ sufficiently close to $t_{0}$. All components except one are intersected with the hyperplane $\Pi$. Therefore, they contain points such that the projection 
of the curve $\Gamma_{t}$ from these points is not convex (the projection of the curve $\Gamma_{t}$ from any point of this hyperplane is not convex since it transversally intersects $\Gamma_{t}$ at $n+2$ points).

The component at infinity (which does not intersect the hyperplane $\Pi$ ) contains such point for $t>t_{0}$ as well. Indeed, the projection $\gamma_{t}$ of the curve $\Gamma_{t}$ from the point $\Theta$ into the hyperplane $\Pi$ is not convex for such $t$ by Proposition 1 (or 3). The component at infinity contains this point for $t<t_{0}$ by Theorem 4 , and, hence, contains it for $t>t_{0}$ by Proposition 8.

Thus, for any $t^{*}>t_{0}$ sufficiently close to $t_{0}$, the curve $\Gamma_{t^{*}}$ has no convex projections from a point lying outside of the curve into any hyperplane (Theorem 4). On the other hand, it is convex by Barner since the curve $\Gamma_{t_{0}}$, as all curves close to it, is convex by Barner according to Theorem 3.

It remains to remark that any curve $\Gamma$ sufficiently close to $\Gamma_{t^{*}}$ is convex by Barner and has no convex projections since the union of osculating hyperplanes to the curve $\Gamma$ at its flattening points is homeomorphic to $\Delta_{t^{*}}$ (by Propositions 2 (or 4), 6 and 7).

6. Properties of curves from Section 3 . Let $\Gamma_{t}$ be the family of curves in $\mathbb{R} P^{2 m+1}$ $=\left\{\left(x_{0}: \ldots: x_{2 m+1}\right)\right\}$ from Section 3 and $\gamma_{t}$ be the projection of the curve $\Gamma_{t}$ from the point $\Theta=(0: \ldots: 0: 1)$ into the hyperplane $\Pi=\left\{x_{2 m+1}=0\right\}$. Consider the lift $\Gamma_{t}^{*}$ of the curve $\Gamma_{t}$ into the affine chart $x_{0}=1$ defined by the functions (1), i.e. $x_{i}=x_{i}(\varphi)$, $i=1, \ldots, 2 m+1$, where

$$
\begin{gathered}
x_{1}(\varphi)=r(\varphi) \cos \varphi, \quad x_{2}(\varphi)=r(\varphi) \sin \varphi, \quad r(\varphi)=1-t \sin ^{2}(m+1) \varphi, \\
x_{3}(\varphi)=\cos 2 \varphi, \quad x_{4}(\varphi)=\sin 2 \varphi, \quad \ldots, \quad x_{2 m-1}(\varphi)=\cos m \varphi, \quad x_{2 m}(\varphi)=\sin m \varphi, \\
x_{2 m+1}(\varphi)=f(\varphi)=\sin \left[(m+1) \varphi+\left(1+(-1)^{m}\right) \frac{\pi}{4}\right], \quad\{\varphi \bmod 2 \pi\} .
\end{gathered}
$$

6.1. Lemmas to the proof of Proposition 1 . For every $\vec{y}=\left(y_{1}, \ldots, y_{2 m+1}\right) \in \mathbb{R}^{2 m+1}$, define the smooth function

$$
F_{\vec{y}, t}(\varphi)=\operatorname{det}\left(\begin{array}{cccc}
x_{1}^{\prime} & \ldots & x_{1}^{(2 m)} & y_{1} \\
\vdots & & \vdots & \vdots \\
x_{2 m+1}^{\prime} & \ldots & x_{2 m+1}^{(2 m)} & y_{2 m+1}
\end{array}\right)
$$

on the curve $\Gamma_{t}^{*}$. For the convenience of its calculation, consider the numbers

$$
\begin{aligned}
\alpha_{1}(k) & =\sum_{i=0}^{k-1}\left(\begin{array}{c}
2 k-1 \\
2 i
\end{array}\right)(2 m+2)^{2 k-2 i-1}, & \alpha_{2}(k) & =\sum_{i=0}^{k-1}\left(\begin{array}{c}
2 k-1 \\
2 i+1
\end{array}\right)(2 m+2)^{2 k-2 i-2}, \\
\beta_{1}(k) & =\sum_{i=0}^{k-1}\left(\begin{array}{c}
2 k \\
2 i+1
\end{array}\right)(2 m+2)^{2 k-2 i-1}, & \beta_{2}(k) & =\sum_{i=0}^{k}\left(\begin{array}{c}
2 k \\
2 i
\end{array}\right)(2 m+2)^{2 k-2 i},
\end{aligned}
$$

and (if $m>1$ ) the matrix

$$
W_{m}=\left(\begin{array}{ccccc}
1 & -2^{2} & 2^{4} & \ldots & (-1)^{m-1} 2^{2 m-2} \\
1 & -3^{2} & 3^{4} & \ldots & (-1)^{m-1} 3^{2 m-2} \\
\vdots & \vdots & \vdots & & \vdots \\
1 & -m^{2} & m^{4} & \ldots & (-1)^{m-1} m^{2 m-2}
\end{array}\right)
$$

Usually, we will omit the subscript $m$ and write $W$. 
Lemma 1.

$$
F_{\vec{y}, t}(\varphi)=\left\{\begin{array}{cccc}
\operatorname{det}\left(\begin{array}{ccc}
a_{1} & b_{1} & \widetilde{y}_{1} \\
a_{2} & b_{2} & \widetilde{y}_{2} \\
f^{\prime}(\varphi) & f^{\prime \prime}(\varphi) & y_{3}
\end{array}\right), & \text { if } m=1, \\
(-1)^{m-1}(m !)^{3} \operatorname{det}\left(\begin{array}{ccc}
A_{1} & B_{1} & \widetilde{y}_{1} \\
A_{2} & B_{2} & \widetilde{y}_{2} \\
W & 0 & Y_{+} \\
0 & W & Y_{-} \\
f^{\prime}(\varphi) C & f^{\prime \prime}(\varphi) C & y_{2 m+1}
\end{array}\right), \quad \text { if } m>1, &
\end{array}\right.
$$

where

$$
Y_{+}=\left(\begin{array}{c}
\frac{1}{2} \widetilde{y}_{4} \\
\vdots \\
\frac{1}{m} \widetilde{y}_{2 m}
\end{array}\right), \quad Y_{-}=\left(\begin{array}{c}
-\frac{1}{2^{2}} \widetilde{y}_{3} \\
\vdots \\
-\frac{1}{m^{2}} \widetilde{y}_{2 m-1}
\end{array}\right)
$$

$\widetilde{y}_{2 i-1}=y_{2 i-1} \cos i \varphi+y_{2 i} \sin i \varphi, \quad \widetilde{y}_{2 i}=-y_{2 i-1} \sin i \varphi+y_{2 i} \cos i \varphi$,

$$
\begin{aligned}
A_{1}=\left(\begin{array}{llll}
a_{1} & a_{3} & \ldots & a_{2 m-1}
\end{array}\right), & B_{1}=\left(\begin{array}{llll}
b_{1} & b_{3} & \ldots & b_{2 m-1}
\end{array}\right), \\
A_{2}=\left(\begin{array}{lllll}
a_{2} & a_{4} & \ldots & a_{2 m}
\end{array}\right), & B_{2}=\left(\begin{array}{lllll}
b_{2} & b_{4} & \ldots & b_{2 m}
\end{array}\right),
\end{aligned}
$$

$a_{2 k-1}=(-1)^{k} \frac{t}{2} \alpha_{1}(k) \sin 2(m+1) \varphi, \quad a_{2 k}=(-1)^{k-1}\left[\left(1-\frac{t}{2}\right)+\frac{t}{2} \alpha_{2}(k) \cos 2(m+1) \varphi\right]$,

$$
\begin{aligned}
& b_{2 k-1}=(-1)^{k}\left[\left(1-\frac{t}{2}\right)+\frac{t}{2} \beta_{2}(k) \cos 2(m+1) \varphi\right], \quad b_{2 k}=(-1)^{k} \frac{t}{2} \beta_{1}(k) \sin 2(m+1) \varphi, \\
& C=\left(\begin{array}{lllll}
1 & -(m+1)^{2} & (m+1)^{4} & \ldots & (-1)^{m-1}(m+1)^{2 m-2}
\end{array}\right) .
\end{aligned}
$$

Proof. Let

$$
u_{2 i-1}^{j}=x_{2 i-1}^{(j)} \cos i \varphi+x_{2 i}^{(j)} \sin i \varphi, \quad u_{2 i}^{j}=-x_{2 i-1}^{(j)} \sin i \varphi+x_{2 i}^{(j)} \cos i \varphi,
$$

$i=1, \ldots, m ; j=1, \ldots, 2 m$. Then

$$
F_{\vec{y}, t}(\varphi)=\operatorname{det}\left(\begin{array}{cccc}
u_{1}^{1} & \ldots & u_{1}^{2 m} & \widetilde{y}_{1} \\
\vdots & & \vdots & \vdots \\
u_{2 m}^{1} & \ldots & u_{2 m}^{2 m} & \widetilde{y}_{2 m} \\
x_{2 m+1}^{\prime} & \ldots & x_{2 m+1}^{(2 m)} & y_{2 m+1}
\end{array}\right) .
$$

Here,

$$
\begin{aligned}
& \left(u_{2 i-1}^{1}, \ldots, u_{2 i-1}^{2 m}\right)=\left(0,-i^{2}, 0, i^{4}, \ldots, 0,(-1)^{m} i^{2 m}\right), \\
& \left(u_{2 i}^{1}, \ldots, u_{2 i}^{2 m}\right)=\left(i, 0,-i^{3}, 0, \ldots,(-1)^{m-1} i^{2 m-1}, 0\right),
\end{aligned}
$$

if $i>1$, and

$$
u_{1}^{j}=\sum_{k=0}^{[j / 2]}(-1)^{k}\left(\begin{array}{c}
j \\
2 k
\end{array}\right) r^{(j-2 k)}, \quad u_{2}^{j}=\sum_{k=0}^{[(j-1) / 2]}(-1)^{k}\left(\begin{array}{c}
j \\
2 k+1
\end{array}\right) r^{(j-2 k-1)},
$$

where $[x]$ is the integral part of the number $x$. It remains only to remark that $u_{1}^{j}=a_{j}$, $u_{2}^{j}=a_{j+1}$, if $j$ is odd, and $u_{1}^{j}=b_{j-1}, u_{2}^{j}=b_{j}$, if $j$ is even. 
Let $W^{k}$ be the determinant of the matrix obtained from $W$ by the deletion of the $k$-th column, if $m>1$, and be 1 , if $m=1$. Consider the smooth function

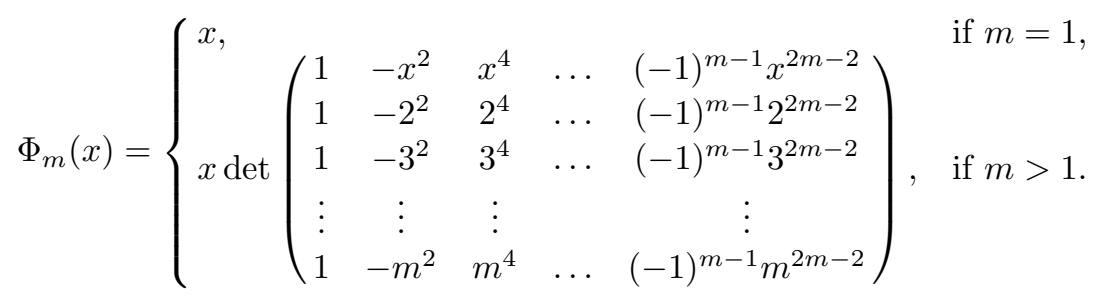

Usually, we will omit the subscript $m$ and write $\Phi(x)$.

LEMMA 2.

$$
\begin{gathered}
\sum_{k=1}^{m} \alpha_{1}(k) W^{k}=\frac{1}{2}[\Phi(2 m+3)+\Phi(2 m+1)], \\
\sum_{k=1}^{m} \alpha_{2}(k) W^{k}=\frac{1}{2}[\Phi(2 m+3)-\Phi(2 m+1)], \\
\sum_{k=1}^{m} \beta_{1}(k) W^{k}=\frac{1}{2}[(2 m+3) \Phi(2 m+3)-(2 m+1) \Phi(2 m+1)], \\
\sum_{k=1}^{m} \beta_{2}(k) W^{k}=\frac{1}{2}[(2 m+3) \Phi(2 m+3)+(2 m+1) \Phi(2 m+1)], \quad \sum_{k=1}^{m} W^{k}=\Phi(1) .
\end{gathered}
$$

This statement follows from the binomial formulas

$$
\begin{aligned}
\alpha_{1}(k)-\alpha_{2}(k)=(2 m+1)^{2 k-1}, & \alpha_{1}(k)+\alpha_{2}(k)=(2 m+3)^{2 k-1}, \\
\beta_{2}(k)-\beta_{1}(k)=(2 m+1)^{2 k}, & \beta_{2}(k)+\beta_{1}(k)=(2 m+3)^{2 k} .
\end{aligned}
$$

and the determinant decomposition theorem.

Let

$$
\begin{gathered}
P=-\frac{t^{2}}{4} \Phi(2 m+1) \Phi(2 m+3), \\
Q=\frac{t}{2}\left(1-\frac{t}{2}\right)[(m+2) \Phi(2 m+3)+m \Phi(2 m+1)] \Phi(1), \\
R=\left(1-\frac{t}{2}\right)^{2} \Phi^{2}(1)+\frac{t^{2}}{16}[\Phi(2 m+3)+\Phi(2 m+1)][(2 m+3) \Phi(2 m+3)-(2 m+1) \Phi(2 m+1)] .
\end{gathered}
$$

LEMma 3. Flattening points of the curve $\gamma_{t}$ are solutions (with the multiplicities taken into account) of the equation

$$
P \cos ^{2} 2(m+1) \varphi+Q \cos 2(m+1) \varphi+R=0 .
$$

Proof. Flattening points of the curve $\gamma_{t}$ are solutions of the equation $F_{\vec{y}, t}(\varphi)=0$, where $\vec{y}=(0, \ldots, 0,1)$. By Lemma 1 , it is equivalent to the equation

$$
\operatorname{det}\left(\begin{array}{ll}
a_{1} & b_{1} \\
a_{2} & b_{2}
\end{array}\right)=0
$$


if $m=1$, and

$$
\operatorname{det}\left(\begin{array}{cc}
A_{1} & B_{1} \\
A_{2} & B_{2} \\
W & 0 \\
0 & W
\end{array}\right)=0,
$$

if $m>1$. Both these equations are equivalent to the equation

$$
\sum_{i, j=1}^{m}(-1)^{i+j} \operatorname{det}\left(\begin{array}{cc}
a_{2 i-1} & b_{2 j-1} \\
a_{2 i} & b_{2 j}
\end{array}\right) W^{i} W^{j}=0
$$

whose left-hand side can be rewritten in the form

$$
\begin{aligned}
& \frac{t^{2}}{4} \sin ^{2} 2(m+1) \varphi \sum_{i=1}^{m} \alpha_{1}(i) W^{i} \sum_{j=1}^{m} \beta_{1}(j) W^{j} \\
& +\frac{t^{2}}{4} \cos ^{2} 2(m+1) \varphi \sum_{i=1}^{m} \alpha_{2}(i) W^{i} \sum_{j=1}^{m} \beta_{2}(j) W^{j} \\
& +\frac{t}{2}\left(1-\frac{t}{2}\right) \cos 2(m+1) \varphi\left[\sum_{i=1}^{m} \alpha_{2}(i) W^{i}+\sum_{j=1}^{m} \beta_{2}(j) W^{j}\right] \sum_{i=1}^{m} W^{i}+\left(1-\frac{t}{2}\right)^{2}\left(\sum_{i=1}^{m} W^{i}\right)^{2}
\end{aligned}
$$

by Lemma 1. Now, Lemma 3 follows from Lemma 2.

LEMMA 4. If $m>1$, then

$$
\Phi(x)=(-1)^{(m-2)(m-1) / 2} \frac{2 x \prod_{i=2}^{m}(2 i-1) !}{(m-1) ! m !(m+1) !} \prod_{i=2}^{m}\left(x^{2}-i^{2}\right) .
$$

The proof is a direct calculation of the Vandermonde determinant which defines the function $\Phi(x)$.

Let us consider the numbers

$$
\begin{gathered}
t_{0}=\frac{4 \Phi(1)}{2 \Phi(1)-(-1)^{m}[(2 m+3) \Phi(2 m+3)+(2 m+1) \Phi(2 m+1)]}, \\
t_{1}=\frac{4 \Phi(1)}{2 \Phi(1)-(-1)^{m}[\Phi(2 m+3)-\Phi(2 m+1)]}
\end{gathered}
$$

LEMMA 5. $0<t_{0}<t_{1}$ and

$$
\frac{1}{t_{0}}=\frac{1}{2}+\frac{(3 m+1) !\left(10 m^{3}+24 m^{2}+15 m+2\right)}{4 m !((m+2) !)^{2}} \geq 9 .
$$

These formulas follow from Lemma 4 (the estimate for $t_{0}$ follows from the fact that $t_{0}$ decreases when $m$ increases).

LEMma 6. The equation

$$
P+(-1)^{m} Q+R=0
$$

is quadratic with respect to $t$ and has two solutions $t_{0}$ and $t_{1}$. 
Proof. The coefficient at $t^{2}$ in this equation is equal to

$$
\begin{aligned}
\frac{1}{16}\left\{2 \Phi(1)-(-1)^{m}[(2 m+3) \Phi(2 m\right. & +3)+(2 m+1) \Phi(2 m+1)]\} \\
\times & \left\{2 \Phi(1)-(-1)^{m}[\Phi(2 m+3)-\Phi(2 m+1)]\right\},
\end{aligned}
$$

and hence, is not equal to 0 by Lemma 4 . The discriminant of the equation is equal to

$$
\frac{1}{4} \Phi^{2}(1)(m+1)^{2}[\Phi(2 m+3)+\Phi(2 m+1)]^{2}
$$

i.e. is a perfect square.

6.2. Proof of Proposition 1. By Lemma 4, the equation from Lemma 3 is quadratic with respect to $\cos 2(m+1) \varphi$ for all $0<t<1$. Its coefficients have the following signs:

$$
P<0, \quad(-1)^{m} Q<0, \quad R>0 .
$$

Since this equation has no solutions for $t=0$ and $P, Q, R$ smoothly depend on $t$, the solutions which can appear when $t$ increases must satisfy equation (2) at the moment of the appearance. Hence, this moment is the smallest positive solution of the equation $P+(-1)^{m} Q+R=0$, less than 1 . By Lemmas 5 and 6 , such a solution exists and is equal to $t_{0}$.

It remains to remark that the expression $P+(-1)^{m} Q+R$ changes its sign when $t$ passes through $t_{0}$. If $t=t_{0}$ and $\varphi_{0}$ is a solution of the equation (2), then the first derivative by $\varphi$ of the left-hand side of the equation from Lemma 3 at $\varphi_{0}$ vanishes, and the second derivative is not zero (it is equal to $\left.-4(m+1)^{2}\left[2 P+(-1)^{m} Q\right]\right)$. Therefore, this equation has $4 m+4$ solutions for any $t>t_{0}$ sufficiently close to $t_{0}$.

6.3. Lemmas to the proof of Proposition $5(n=2 m)$. Let

$$
\begin{array}{ccc}
A_{2}^{0}=\left(\begin{array}{llll}
a_{2}^{0} & a_{4}^{0} & \ldots & a_{2 m}^{0}
\end{array}\right), & a_{2 k}^{0}=(-1)^{k-1}\left[\left(1-\frac{t}{2}\right)+(-1)^{m} \frac{t}{2} \alpha_{2}(k)\right], \\
B_{1}^{0}=\left(\begin{array}{llll}
b_{1}^{0} & b_{3}^{0} & \ldots & b_{2 m-1}^{0}
\end{array}\right), & b_{2 k-1}^{0}=(-1)^{k}\left[\left(1-\frac{t}{2}\right)+(-1)^{m} \frac{t}{2} \beta_{2}(k)\right] .
\end{array}
$$

LEMMA 7. If $m>1$, then

$$
\begin{aligned}
& \operatorname{det}\left(\begin{array}{l}
A_{2}^{0} \\
W
\end{array}\right)=\Phi(1)\left(1-\frac{t}{t_{1}}\right), \quad \operatorname{det}\left(\begin{array}{c}
B_{1}^{0} \\
W
\end{array}\right)=-\Phi(1)\left(1-\frac{t}{t_{0}}\right), \\
& \operatorname{det}\left(\begin{array}{c}
W \\
C
\end{array}\right)=(-1)^{m-1} \frac{\Phi(m+1)}{m+1} \text {. }
\end{aligned}
$$

Proof. By Lemma 2,

$$
\begin{aligned}
\operatorname{det}\left(\begin{array}{l}
A_{2}^{0} \\
W
\end{array}\right) & =\left(1-\frac{t}{2}\right) \Phi(1)+(-1)^{m} \frac{t}{4}[\Phi(2 m+3)-\Phi(2 m+1)]=\Phi(1)\left(1-\frac{t}{t_{1}}\right), \\
\operatorname{det}\left(\begin{array}{c}
B_{1}^{0} \\
W
\end{array}\right) & =-\left(1-\frac{t}{2}\right) \Phi(1) \\
& -(-1)^{m} \frac{t}{4}[(2 m+3) \Phi(2 m+3)+(2 m+1) \Phi(2 m+1)]=-\Phi(1)\left(1-\frac{t}{t_{0}}\right) .
\end{aligned}
$$

The third equality is evident. 
LEMMA 8. Let $\varphi_{0}$ be a solution of equation (2). Then

$$
\begin{array}{ll}
F_{\vec{y}, t}\left(\varphi_{0}\right)=(1-t)\left[f^{\prime \prime}\left(\varphi_{0}\right)\left(y_{1} \cos \varphi_{0}+y_{2} \sin \varphi_{0}\right)+(1-9 t) y_{3}\right], & \text { if } m=1, \\
F_{\vec{y}, t}\left(\varphi_{0}\right)=\Phi(1)(m !)^{3}\left(1-\frac{t}{t_{1}}\right)\left\{f ^ { \prime \prime } ( \varphi _ { 0 } ) \left[\frac{\Phi(m+1)}{m+1}\left(y_{1} \cos \varphi_{0}+y_{2} \sin \varphi_{0}\right)\right.\right. & \\
\left.\left.\quad+\sum_{i=2}^{m} \frac{y_{2 i-1} \cos i \varphi_{0}+y_{2 i} \sin i \varphi_{0}}{i^{2}} \operatorname{det}\left(\begin{array}{cc}
B_{1}^{0} & 0 \\
W & e_{i} \\
C & 0
\end{array}\right)\right]+\Phi(1)\left(1-\frac{t}{t_{0}}\right) y_{2 m+1}\right\}, & \text { if } m>1,
\end{array}
$$

where $e_{i}$ is the $(m-1)$-element column whose $(i-1)$-th element is equal to 1 , and all other are equal to 0 .

Proof. By Lemma 1

$$
F_{\vec{y}, t}\left(\varphi_{0}\right)=\left\{\begin{array}{ccc}
\operatorname{det}\left(\begin{array}{ccc}
0 & b_{1}^{0} & \widetilde{y}_{1} \\
a_{2}^{0} & 0 & \widetilde{y}_{2} \\
0 & f^{\prime \prime}\left(\varphi_{0}\right) & y_{3}
\end{array}\right), & & \text { if } m=1, \\
(-1)^{m-1}(m !)^{3} \operatorname{det}\left(\begin{array}{ccc}
0 & B_{1}^{0} & \widetilde{y}_{1} \\
A_{2}^{0} & 0 & \widetilde{y}_{2} \\
W & 0 & Y_{+} \\
0 & W & Y_{-} \\
0 & f^{\prime \prime}\left(\varphi_{0}\right) C & y_{2 m+1}
\end{array}\right), \quad \text { if } m>1 .
\end{array}\right.
$$

Therefore, $F_{\vec{y}, t}\left(\varphi_{0}\right)=a_{2}^{0}\left[\widetilde{y}_{1} f^{\prime \prime}\left(\varphi_{0}\right)-b_{1}^{0} y_{3}\right]$, if $m=1$, and

$$
F_{\vec{y}, t}\left(\varphi_{0}\right)=(m !)^{3} \operatorname{det}\left(\begin{array}{c}
A_{2}^{0} \\
W
\end{array}\right)\left[(-1)^{m-1} \widetilde{y}_{1} f^{\prime \prime}\left(\varphi_{0}\right) \operatorname{det}\left(\begin{array}{c}
W \\
C
\end{array}\right)-\operatorname{det}\left(\begin{array}{cc}
B_{1}^{0} & 0 \\
W & Y_{-} \\
f^{\prime \prime}\left(\varphi_{0}\right) C & y_{2 m+1}
\end{array}\right)\right] \text {, }
$$

if $m>1$. Now, Lemma 8 follows from Lemma 7 .

6.4. Proof of Proposition $5(n=2 m)$. Let $\varphi_{0}$ be a flattening point of the curve $\gamma_{t_{0}}$. Then the function $F_{\vec{y}, t_{0}}\left(\varphi_{0}\right)$ of the variable $\vec{y}$ is not equal to 0 identically by Proposition 1 , Lemmas 5 and 8 . Hence, the vectors of the leading $2 m$ derivatives of the curve $\Gamma_{t_{0}}^{*}$ at the point $\varphi_{0}$ are linearly independent.

Now, consider the vectors of the leading $2 m-1$ derivatives of the curve $\gamma_{t}$ at the point $\varphi_{0}$. By Lemma 1, the rank of the system of these vectors is equal to the rank of the system of the leading $2 m-1$ columns of the matrix

$$
\Lambda= \begin{cases}\left(\begin{array}{cc}
0 & b_{1}^{0} \\
a_{2}^{0} & 0
\end{array}\right), & \text { if } m=1, \\
\left(\begin{array}{cc}
0 & B_{1}^{0} \\
A_{2}^{0} & 0 \\
W & 0 \\
0 & W
\end{array}\right), & \text { if } m>1 .\end{cases}
$$

The determinant of the matrix obtained from $\Lambda$ by the deletion of the first line and the last column is equal to

$$
\chi= \begin{cases}a_{2}^{0}, & \text { if } m=1, \\
\operatorname{det}\left(\begin{array}{l}
A_{2}^{0} \\
W
\end{array}\right) \operatorname{det} W^{m}, & \text { if } m>1,\end{cases}
$$


where $a_{2}^{0}=1-t$ (if $\left.m=1\right)$ and $\operatorname{det} W^{m} \neq 0$ (the Vandermonde determinant). According to Lemmas 5 and $7, \chi \neq 0$ for $t=t_{0}$. Hence, the vectors of the leading $2 m-1$ derivatives of the curve $\gamma_{t_{0}}$ at the point $\varphi_{0}$ are linearly independent.

6.5. Lemmas to the proof of Proposition 2. Let

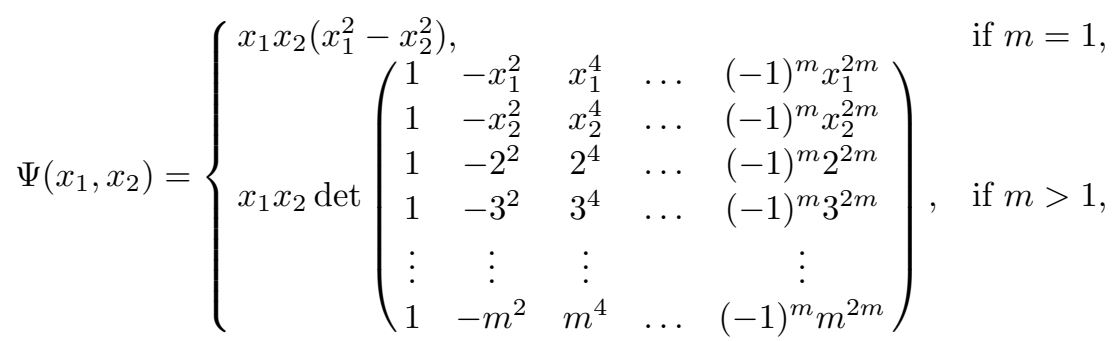

$\widetilde{P}=-\frac{t^{2}}{8}\left[(2 m+3) \Phi_{m+1}(2 m+1) \Phi(2 m+3)\right.$

$\left.-(2 m+1) \Phi_{m+1}(2 m+3) \Phi(2 m+1)+(-1)^{m} \Phi(m+1) \Psi(2 m+1,2 m+3)\right]$,

$\widetilde{Q}=\frac{t}{4}\left(1-\frac{t}{2}\right)\left\{\Phi_{m+1}(1)[(2 m+3) \Phi(2 m+3)+(2 m+1) \Phi(2 m+1)]\right.$

$-(-1)^{m} \Phi(m+1)[\Psi(2 m+3,1)+\Psi(2 m+1,1)]+\Phi(1)\left[\Phi_{m+1}(2 m+3)\right.$

$$
\left.\left.-\Phi_{m+1}(2 m+1)\right]\right\}-\frac{t^{2}}{8} \Phi(m+1) \Psi(2 m+1,2 m+3),
$$

$\widetilde{R}=\left(1-\frac{t}{2}\right)^{2} \Phi_{m+1}(1) \Phi(1)+\frac{t^{2}}{16}\left[\Phi_{m+1}(2 m+3)\right.$

$$
\left.+\Phi_{m+1}(2 m+1)\right][(2 m+3) \Phi(2 m+3)-(2 m+1) \Phi(2 m+1)]
$$

$$
-\frac{t}{4}\left(1-\frac{t}{2}\right) \Phi(m+1)[\Psi(2 m+1,1)+\Psi(2 m+3,1)] .
$$

Lemma 9. Flattening points of the curve $\Gamma_{t}$ are solutions (with the multiplicities taken into account) of the equation

$$
f^{\prime}(\varphi)\left[\widetilde{P} \cos ^{2} 2(m+1) \varphi+\widetilde{Q} \cos 2(m+1) \varphi+\widetilde{R}\right]=0 .
$$

Proof. These points are solutions of the equation $F_{\vec{y}, t}(\varphi)=0$ where

$$
\vec{y}=\left(x_{1}^{(2 m+1)}, \ldots, x_{2 m+1}^{(2 m+1)}\right) .
$$

By Lemma 1, it is equivalent to the equation

$$
\operatorname{det}\left(\begin{array}{ccc}
a_{1} & b_{1} & a_{3} \\
a_{2} & b_{2} & a_{4} \\
f^{\prime}(\varphi) & f^{\prime \prime}(\varphi) & y_{3}
\end{array}\right)=0
$$

if $m=1$, and

$$
\operatorname{det}\left(\begin{array}{ccc}
A_{1} & B_{1} & a_{2 m+1} \\
A_{2} & B_{2} & a_{2 m+2} \\
W & 0 & Y_{+} \\
0 & W & 0 \\
f^{\prime}(\varphi) C & f^{\prime \prime}(\varphi) C & y_{2 m+1}
\end{array}\right)=0,
$$


if $m>1$, where

$$
Y_{+}=(-1)^{m}\left(\begin{array}{c}
2^{2 m} \\
\vdots \\
m^{2 m}
\end{array}\right), \quad y_{2 m+1}=(-1)^{m}(m+1)^{2 m} f^{\prime}(\varphi) .
$$

The left-hand sides of the indicated equations are equal to

$$
\begin{gathered}
f^{\prime}(\varphi) \operatorname{det}\left(\begin{array}{ccc}
a_{1} & b_{1} & a_{3} \\
a_{2} & b_{2} & a_{4} \\
1 & 0 & -4
\end{array}\right)+f^{\prime \prime}(\varphi) \operatorname{det}\left(\begin{array}{ccc}
a_{1} & b_{1} & a_{3} \\
a_{2} & b_{2} & a_{4} \\
0 & 1 & 0
\end{array}\right) \\
\quad=-f^{\prime}(\varphi)\left[\operatorname{det}\left(\begin{array}{c}
\widetilde{A}_{2} \\
W_{2}
\end{array}\right) b_{1}-\operatorname{det}\left(\begin{array}{c}
\widetilde{A}_{1} \\
W_{2}
\end{array}\right) b_{2}\right]-f^{\prime \prime}(\varphi) \operatorname{det}\left(\begin{array}{cc}
a_{1} & a_{3} \\
a_{2} & a_{4}
\end{array}\right)
\end{gathered}
$$

and

$$
\begin{array}{r}
f^{\prime}(\varphi) \operatorname{det}\left(\begin{array}{ccc}
A_{1} & B_{1} & a_{2 m+1} \\
A_{2} & B_{2} & a_{2 m+2} \\
W & 0 & Y_{+} \\
0 & W & 0 \\
C & 0 & (-1)^{m}(m+1)^{2 m}
\end{array}\right)+f^{\prime \prime}(\varphi) \operatorname{det}\left(\begin{array}{ccc}
A_{1} & B_{1} & a_{2 m+1} \\
A_{2} & B_{2} & a_{2 m+2} \\
W & 0 & Y_{+} \\
0 & W & 0 \\
0 & C & 0
\end{array}\right) \\
=(-1)^{m} f^{\prime}(\varphi)\left[\operatorname{det}\left(\begin{array}{c}
\widetilde{A}_{2} \\
W_{m+1}
\end{array}\right) \operatorname{det}\left(\begin{array}{c}
B_{1} \\
W
\end{array}\right)-\operatorname{det}\left(\begin{array}{c}
\widetilde{A}_{1} \\
W_{m+1}
\end{array}\right) \operatorname{det}\left(\begin{array}{c}
B_{2} \\
W
\end{array}\right)\right] \\
+(-1)^{m} f^{\prime \prime}(\varphi) \operatorname{det}\left(\begin{array}{cc}
A_{1} & a_{2 m+1} \\
A_{2} & a_{2 m+2} \\
W & Y_{+}
\end{array}\right) \operatorname{det}\left(\begin{array}{c}
W \\
C
\end{array}\right),
\end{array}
$$

respectively, where

$$
\widetilde{A}_{1}=\left(\begin{array}{llll}
a_{1} & a_{3} & \ldots & a_{2 m+1}
\end{array}\right), \quad \widetilde{A}_{2}=\left(\begin{array}{llll}
a_{2} & a_{4} & \ldots & a_{2 m+2}
\end{array}\right) .
$$

By Lemma 2 and the definition of the function $\Psi$, we have:

$$
\begin{gathered}
\operatorname{det}\left(\begin{array}{c}
\widetilde{A}_{1} \\
W_{m+1}
\end{array}\right)=-\frac{t}{4}\left[\Phi_{m+1}(2 m+3)+\Phi_{m+1}(2 m+1)\right] \sin 2(m+1) \varphi, \\
\operatorname{det}\left(\begin{array}{c}
\widetilde{A}_{2} \\
W_{m+1}
\end{array}\right)=\left(1-\frac{t}{2}\right) \Phi_{m+1}(1)+\frac{t}{4}\left[\Phi_{m+1}(2 m+3)-\Phi_{m+1}(2 m+1)\right] \cos 2(m+1) \varphi, \\
\operatorname{det}\left(\begin{array}{c}
B_{1} \\
W
\end{array}\right)=-\left(1-\frac{t}{2}\right) \Phi(1)-\frac{t}{4}[(2 m+3) \Phi(2 m+3)+(2 m+1) \Phi(2 m+1)] \cos 2(m+1) \varphi, \\
\operatorname{det}\left(\begin{array}{c}
B_{2} \\
W
\end{array}\right)=-\frac{t}{4}[(2 m+3) \Phi(2 m+3)-(2 m+1) \Phi(2 m+1)] \sin 2(m+1) \varphi, \\
\operatorname{det}\left(\begin{array}{cc}
A_{1} & a_{2 m+1} \\
A_{2} & a_{2 m+2} \\
W & Y_{+}
\end{array}\right)=-\frac{t}{4} \sin 2(m+1) \varphi \\
\times\left\{\begin{array}{cc}
\left.\left(1-\frac{t}{2}\right)[\Psi(2 m+1,1)+\Psi(2 m+3,1)]+\frac{t}{2} \Psi(2 m+1,2 m+3) \cos 2(m+1) \varphi\right\},
\end{array}\right.
\end{gathered}
$$

These formulas and Lemma 7 imply the above equation for flattening points of the curve $\Gamma_{t}$. 
Lemma 10. If $m>1$, then

$$
\Psi\left(x_{1}, x_{2}\right)=(-1)^{(m-2)(m-1) / 2} \frac{2 x_{1} x_{2}\left(x_{1}^{2}-x_{2}^{2}\right) \prod_{i=2}^{m}(2 i-1) !}{(m-1) ! m !(m+1) !} \prod_{i=2}^{m}\left(x_{1}^{2}-i^{2}\right) \prod_{i=2}^{m}\left(x_{2}^{2}-i^{2}\right) .
$$

The proof is a direct calculation of the Vandermonde determinant which defines the function $\Psi\left(x_{1}, x_{2}\right)$.

Lemma 11. $(-1)^{m} \widetilde{P}<0$ for any $t \neq 0$.

Proof. By Lemmas 4 and 10,

$$
\begin{aligned}
\widetilde{P}=(-1)^{m-1} t^{2}\left(\frac{\prod_{i=2}^{m}(2 i-1) !}{(m-1) ! m !(m+1) !}\right)^{2} \frac{(2 m+1)(2 m+3)(2 m+1) !}{(m+1)} & \\
& \times \prod_{i=2}^{m}\left((2 m+1)^{2}-i^{2}\right) \prod_{i=2}^{m}\left((2 m+3)^{2}-i^{2}\right),
\end{aligned}
$$

if $m>1$, and $\widetilde{P}=\frac{45}{4} t^{2}$, if $m=1$.

Lemma 12. $\widetilde{Q}>0$ for any $0<t<2$.

P r o of. Lemmas 4 and 10 imply:

$$
\begin{gathered}
(2 m+1) \Phi_{m+1}(1) \Phi(2 m+1)-(-1)^{m} \Phi(m+1) \Psi(2 m+1,1)>0, \\
(2 m+3) \Phi_{m+1}(1) \Phi(2 m+3)-(-1)^{m} \Phi(m+1) \Psi(2 m+3,1)>0, \\
\Phi(1)\left[\Phi_{m+1}(2 m+3)-\Phi_{m+1}(2 m+1)\right]>0, \quad \Phi(m+1) \Psi(2 m+1,2 m+3)<0 .
\end{gathered}
$$

Therefore $\widetilde{Q}>0$ if $t$ and $1-t / 2$ are positive.

The following statement is evident.

Lemma 13. $(-1)^{m} \widetilde{R}>0$ for any $t$ sufficiently close to 0 .

Let us consider the numbers

and

$$
T_{0}=\frac{4 \Phi_{m+1}(1)}{2 \Phi_{m+1}(1)+(-1)^{m}\left[\Phi_{m+1}(2 m+3)-\Phi_{m+1}(2 m+1)\right]}
$$

$$
T_{1}=\frac{4 \Phi(1)}{2 \Phi(1)+(-1)^{m}[(2 m+3) \Phi(2 m+3)+(2 m+1) \Phi(2 m+1)]} .
$$

LEMMA 14. $T_{1}<0, T_{0}>t_{0}$ and

$$
\frac{1}{T_{0}}=\frac{1}{2}+\frac{(3 m+2) !(4 m+5)}{4 m !((m+2) !)^{2}} \geq 8
$$

These formulas follow from Lemmas 4 and 5 .

LEMma 15. The equation

$$
\widetilde{P}+(-1)^{m-1} \widetilde{Q}+\widetilde{R}=0
$$

is quadratic with respect to $t$ and has two solutions $T_{0}$ and $T_{1}$. 
Proof. The coefficient at $t^{2}$ in this equation is equal to

$$
\begin{aligned}
\frac{1}{16}\left\{2 \Phi(1)+(-1)^{m}[(2 m\right. & +3) \Phi(2 m+3)+(2 m+1) \Phi(2 m+1)]\} \\
\times & \left\{2 \Phi_{m+1}(1)+(-1)^{m}\left[\Phi_{m+1}(2 m+3)-\Phi_{m+1}(2 m+1)\right]\right\},
\end{aligned}
$$

and hence, is not equal to 0 by Lemma 4 . The discriminant of the equation equals

$$
\begin{aligned}
\frac{1}{16}\left\{\Phi_{m+1}(1)[(2 m+3) \Phi(2 m+3)+\right. & (2 m+1) \Phi(2 m+1)] \\
& \left.-\Phi(1)\left[\Phi_{m+1}(2 m+3)-\Phi_{m+1}(2 m+1)\right]\right\}^{2},
\end{aligned}
$$

i.e. is a perfect square.

6.6. Proof of Proposition 2. By Lemma 9, flattening points of the curve $\Gamma_{t}$ are defined by the equations $f^{\prime}(\varphi)=0$ and

$$
\widetilde{P} \cos ^{2} 2(m+1) \varphi+\widetilde{Q} \cos 2(m+1) \varphi+\widetilde{R}=0 .
$$

The first one has $2 m+2$ solutions $\cos 2(m+1) \varphi=(-1)^{m}$. The second one has no solutions if $t=0$ and is quadratic with respect to $\cos 2(m+1) \varphi$ for every $t \neq 0$.

Since $\widetilde{P}, \widetilde{Q}, \widetilde{R}$ smoothly depend on $t$ and satisfy the inequalities from Lemmas 11-13, the solutions which can appear from the second equation when $t$ increases from 0 to 1 must satisfy the equation $\cos 2(m+1) \varphi=(-1)^{m-1}$ at the moment of the appearance. Hence, this moment is the smallest positive solution of the equation $\widetilde{P}+(-1)^{m-1} \widetilde{Q}+\widetilde{R}=0$, less than 1. By Lemmas 14 and 15, such a solution exists, is equal to $T_{0}$ and greater than $t_{0}$.

It remains to remark that for any $t \in\left[0, T_{0}\right)$ and any solution $\varphi_{0}$ of equation $(2)$, the first derivative by $\varphi$ of the left-hand side of the equation from Lemma 9 at $\varphi_{0}$ is not equal to 0 .

6.7. Lemmas to the proofs of Propositions 6-8 $(n=2 m)$. The osculating hyperplane to the curve $\Gamma_{t}$ at a point $\varphi$ is defined by the equation

$$
F_{\vec{y}, t}(\varphi)=y_{0} F_{\Gamma_{t}^{*}(\varphi), t}(\varphi),
$$

where $\vec{y}=\left(y_{1}, \ldots, y_{2 m+1}\right)$ and $\left(y_{0}: y_{1}: \ldots: y_{2 m+1}\right) \in \mathbb{R} P^{2 m+1}$.

Lemma 16. Let $\varphi_{0}$ be a solution of equation $(2)$. Then $F_{\Gamma_{t_{0}}^{*}\left(\varphi_{0}\right), t_{0}}\left(\varphi_{0}\right) \neq 0$.

Proof. The equation $F_{\vec{y}, t_{0}}\left(\varphi_{0}\right)=0$ defines a hyperplane in $\Pi \cong \mathbb{R} P^{2 m}$ by Lemma 8 . This hyperplane intersects the curve $\gamma_{t_{0}}$ at least at two geometrically different points by Sturm-Hurwitz theorem $[\mathrm{H}]$. Therefore, it cannot be the osculating hyperplane to the curve $\gamma_{t_{0}}$ at any of its multiple flattening point by Remark 11 and Theorem 2.

LEMma 17. If $t$ is sufficiently close to $t_{0}$, then the determinant

$$
\operatorname{det}\left(\begin{array}{cc}
B_{1}^{0} & 0 \\
W & e_{i} \\
C & 0
\end{array}\right)
$$

from Lemma 8 is not equal to 0 for any $i=2, \ldots, m$. 
Proof. Let us consider the more general determinant

$$
\operatorname{det}\left(\begin{array}{cc}
B_{1}^{0} & 0 \\
W & \delta_{i}^{*} \\
C & \delta_{i}^{m+1}
\end{array}\right), \quad i \in\{2, \ldots, m+1\},
$$

where the $i$-th element of the last column equals to 1 , and all other equal 0 . By the Vandermonde formula, this determinant is equal to

$$
\begin{aligned}
& (-1)^{i} \prod_{\substack{2 \leq k<l \leq m+1 \\
k \neq i, l \neq i}}\left(k^{2}-l^{2}\right)\left\{-\left(1-\frac{t}{2}\right) \frac{1}{i^{2}-1} \prod_{j=2}^{m+1}\left(j^{2}-1\right)\right. \\
& \left.+\frac{t}{4}\left[\frac{(2 m+1)^{2}}{(2 m+1)^{2}-i^{2}} \prod_{j=2}^{m+1}\left((2 m+1)^{2}-j^{2}\right)+\frac{(2 m+3)^{2}}{(2 m+3)^{2}-i^{2}} \prod_{j=2}^{m+1}\left((2 m+3)^{2}-j^{2}\right)\right]\right\} .
\end{aligned}
$$

This expression vanishes for $t=\tau_{i}(m)$ where

$$
\begin{aligned}
\frac{1}{\tau_{i}(m)}=\frac{1}{2}+\frac{1}{4 \prod_{j=2}^{m+1}\left(j^{2}-1\right)} & {\left[\frac{i^{2}-1}{(2 m+1)^{2}-i^{2}}(2 m+1)^{2} \prod_{j=2}^{m+1}\left((2 m+1)^{2}-j^{2}\right)\right.} \\
& \left.+\frac{i^{2}-1}{(2 m+3)^{2}-i^{2}}(2 m+3)^{2} \prod_{j=2}^{m+1}\left((2 m+3)^{2}-j^{2}\right)\right] .
\end{aligned}
$$

It is evident that $\tau_{2}(m)>\ldots>\tau_{m}(m)>\tau_{m+1}(m)$ since the function $\frac{x-1}{\lambda-x}$ increases on the interval $x \in(-\infty, \lambda)$ for any $\lambda>1$. It remains to remark that $\tau_{m+1}(m)=t_{0}$ by Lemma 7.

Remark 12. The moments $\tau_{i}(m), i=2, \ldots, m$, as well as the moments $t_{0}, T_{0}$, define some bifurcations in the family $\Gamma_{t}$. In particular, the beginning of the sequence $\tau_{m}(m)$ for $m>1$ is:

$$
\frac{4}{297}, \quad \frac{1}{2657}, \quad \frac{4}{307925}, \quad \frac{1}{2082152}, \quad \frac{4}{218972327}, \quad \ldots
$$

It is easy to see that $\tau_{m}(m)<T_{0}=T_{0}(m)$ already for $m=3$.

6.8. Proof of Proposition $6(n=2 m)$. Let $\varphi_{1}, \ldots, \varphi_{2 m+1}$ be mutually different solutions of equation (2). Then $\Theta$ is a unique solution of the linear equations system

$$
F_{\vec{y}, t_{0}}\left(\varphi_{i}\right)=y_{0} F_{\Gamma_{t_{0}}^{*}\left(\varphi_{i}\right), t_{0}}\left(\varphi_{i}\right), \quad i=1, \ldots, 2 m+1 .
$$

Indeed, the point $\Theta$ satisfies this system by Lemma 8 . The rank of the system is equal to the rank of the matrix formed by the coordinates of the vectors

$$
\left(1, \cos \varphi_{i}, \sin \varphi_{i}, \cos 2 \varphi_{i}, \sin 2 \varphi_{i}, \ldots, \cos m \varphi_{i}, \sin m \varphi_{i}\right), \quad i=1, \ldots, 2 m+1,
$$

(this follows from Lemmas 4, 5, 8, 16 and 17). But the determinant of this matrix is not 0 (even for any mutually different points $\varphi_{1}, \ldots, \varphi_{2 m+1}$ on the circle $\{\varphi \bmod 2 \pi\}$ ) since the curve $\gamma_{0}$ is convex. 
6.9. Proof of Proposition $7(n=2 m)$. Let us consider the solutions

$$
\varphi_{i}=\frac{1-(-1)^{m}}{4(m+1)} \pi+\frac{\pi}{m+1} i, \quad i=0,1, \ldots, 2 m+1,
$$

of equation (2). The intersection of the osculating hyperplanes to the curve $\Gamma_{t}$ at these points is defined by the linear equations system

$$
F_{\vec{y}, t}\left(\varphi_{i}\right)=y_{0} F_{\Gamma_{t}^{*}\left(\varphi_{i}\right), t}\left(\varphi_{i}\right), \quad i=0,1, \ldots, 2 m+1 .
$$

If $t$ is sufficiently close to $t_{0}$ and $t \neq t_{0}$, then by Lemmas $4,5,8,16$ and 17 , the rank of this system is equal to the rank of the matrix formed by the coordinates of the vectors

$$
\begin{array}{rl}
v_{i}=\left(1, \cos \varphi_{i}, \sin \varphi_{i}, \cos 2 \varphi_{i}, \sin 2 \varphi_{i}, \ldots, \cos m \varphi_{i}, \sin m \varphi_{i}, f\left(\varphi_{i}\right)\right) & \\
i & i=0,1, \ldots, 2 m+1 .
\end{array}
$$

Let us suppose that these vectors lie in a hyperplane in $\mathbb{R}^{2 m+2}$. Then this hyperplane contains the vertical vector $(0, \ldots, 0,1)$ since

$$
\frac{1}{m+1} \sum_{k=0}^{m} v_{2 k}=(1,0, \ldots, 0,1), \quad \frac{1}{m+1} \sum_{k=0}^{m} v_{2 k+1}=(1,0, \ldots, 0,-1) .
$$

Hence, the vectors

$$
\widetilde{v}_{i}=\left(1, \cos \varphi_{i}, \sin \varphi_{i}, \cos 2 \varphi_{i}, \sin 2 \varphi_{i}, \ldots, \cos m \varphi_{i}, \sin m \varphi_{i}\right), \quad i=0,1, \ldots, 2 m+1,
$$

lie in a hyperplane in $\mathbb{R}^{2 m+1}$. But this contradicts the convexity of the curve $\gamma_{0}$.

6.10. Proof of Proposition $8(n=2 m)$. Let $\vec{y}=(0, \ldots, 0,1) \in \mathbb{R}^{2 m+1}$ and $\varphi$ be a solution of equation (2). Then

$$
F_{\vec{y}, t}(\varphi)=\Phi^{2}(1)(m !)^{3}\left(1-\frac{t}{t_{1}}\right)\left(1-\frac{t}{t_{0}}\right)
$$

by Lemma 8 . Now, the statement follows from Lemmas 4 and 5 .

7. Properties of curves from Section 4. Let $\Gamma_{t}$ be the family of curves in $\mathbb{R} P^{2 m}=$ $\left\{\left(x_{1}: \ldots: x_{2 m+1}\right)\right\}$ from Section 4 and $\gamma_{t}$ be the projection of the curve $\Gamma_{t}$ from the point $\Theta=(0: \ldots: 0: 1)$ into the hyperplane $\Pi=\left\{x_{2 m+1}=0\right\}$. Consider the double covering $\Gamma_{t}^{*}$ of the curve $\Gamma_{t}$ in the space $\mathbb{R}^{2 m+1}$ defined by the functions $(3)$, i.e. $x_{i}=x_{i}(\varphi)$, $i=1, \ldots, 2 m+1$, where

$$
\begin{gathered}
x_{1}(\varphi)=\cos \varphi-t \cos (4 m+3) \varphi, \quad x_{2}(\varphi)=\sin \varphi-t \sin (4 m+3) \varphi, \\
x_{3}(\varphi)=\cos 3 \varphi, x_{4}(\varphi)=\sin 3 \varphi, \ldots, x_{2 m-1}(\varphi)=\cos (2 m-1) \varphi, x_{2 m}(\varphi)=\sin (2 m-1) \varphi, \\
x_{2 m+1}(\varphi)=f(\varphi)=\sin \left[(2 m+1) \varphi+\left(1+(-1)^{m}\right) \frac{\pi}{4}\right], \quad\{\varphi \bmod 2 \pi\} .
\end{gathered}
$$

7.1. Lemmas to the proof of Proposition 3. For every $\vec{y}=\left(y_{1}, \ldots, y_{2 m+1}\right) \in \mathbb{R}^{2 m+1}$, let us define the smooth function

$$
F_{\vec{y}, t}(\varphi)=\operatorname{det}\left(\begin{array}{ccccc}
x_{1} & x_{1}^{\prime} & \ldots & x_{1}^{(2 m-1)} & y_{1} \\
\vdots & \vdots & & \vdots & \vdots \\
x_{2 m+1} & x_{2 m+1}^{\prime} & \ldots & x_{2 m+1}^{(2 m-1)} & y_{2 m+1}
\end{array}\right)
$$


on the curve $\Gamma_{t}^{*}$. For the convenience of its calculation in the case $m>1$, consider the matrix

$$
W_{m}=\left(\begin{array}{ccccc}
1 & -3^{2} & 3^{4} & \ldots & (-1)^{m-1} 3^{2 m-2} \\
1 & -5^{2} & 5^{4} & \ldots & (-1)^{m-1} 5^{2 m-2} \\
\vdots & \vdots & \vdots & & \vdots \\
1 & -(2 m-1)^{2} & (2 m-1)^{4} & \ldots & (-1)^{m-1}(2 m-1)^{2 m-2}
\end{array}\right) .
$$

Usually, we will omit the subscript $m$ and write $W$.

Lemma 18.

$$
F_{\vec{y}, t}(\varphi)=\left\{\begin{array}{ccc}
\operatorname{det}\left(\begin{array}{ccc}
a_{0} & a_{1} & \widetilde{y}_{1} \\
b_{0} & b_{1} & \widetilde{y}_{2} \\
f(\varphi) & f^{\prime}(\varphi) & y_{3}
\end{array}\right), & & \text { if } m=1, \\
(-1)^{m-1}(2 m-1) ! ! \operatorname{det}\left(\begin{array}{ccc}
A_{0} & A_{1} & \widetilde{y}_{1} \\
B_{0} & B_{1} & \widetilde{y}_{2} \\
W & 0 & Y_{+} \\
0 & W & Y_{-} \\
f(\varphi) C & f^{\prime}(\varphi) C & y_{2 m+1}
\end{array}\right), & \text { if } m>1,
\end{array}\right.
$$

where

$$
\begin{gathered}
Y_{+}=\left(\begin{array}{c}
\widetilde{y}_{3} \\
\vdots \\
\widetilde{y}_{2 m-1}
\end{array}\right), \quad Y_{-}=\left(\begin{array}{c}
\frac{1}{3} \widetilde{y}_{4} \\
\vdots \\
\frac{1}{2 m-1} \widetilde{y}_{2 m}
\end{array}\right), \\
\widetilde{y}_{2 i-1}=y_{2 i-1} \cos (2 i-1) \varphi+y_{2 i} \sin (2 i-1) \varphi, \\
\widetilde{y}_{2 i}=-y_{2 i-1} \sin (2 i-1) \varphi+y_{2 i} \cos (2 i-1) \varphi,
\end{gathered}
$$

$$
\begin{aligned}
& A_{0}=\left(\begin{array}{llll}
a_{0} & a_{2} & \ldots & a_{2 m-2}
\end{array}\right), a_{2 k}=(-1)^{k}\left[1-(4 m+3)^{2 k} t \cos (4 m+2) \varphi\right], \\
& A_{1}=\left(\begin{array}{llll}
a_{1} & a_{3} & \ldots & a_{2 m-1}
\end{array}\right), a_{2 k+1}=(-1)^{k}(4 m+3)^{2 k+1} t \sin (4 m+2) \varphi, \\
& B_{0}=\left(\begin{array}{llll}
b_{0} & b_{2} & \ldots & b_{2 m-2}
\end{array}\right), b_{2 k}=(-1)^{k+1}(4 m+3)^{2 k} t \sin (4 m+2) \varphi, \\
& B_{1}=\left(\begin{array}{lllll}
b_{1} & b_{3} & \ldots & b_{2 m-1}
\end{array}\right), b_{2 k+1}=(-1)^{k}\left[1-(4 m+3)^{2 k+1} t \cos (4 m+2) \varphi\right], \\
& C=\left(\begin{array}{lllll}
1 & -(2 m+1)^{2} & (2 m+1)^{4} & \ldots & \left.(-1)^{m-1}(2 m+1)^{2 m-2}\right) .
\end{array}\right.
\end{aligned}
$$

Proof. Let

$$
\begin{aligned}
& u_{2 i-1}^{j}=x_{2 i-1}^{(j)} \cos (2 i-1) \varphi+x_{2 i}^{(j)} \sin (2 i-1) \varphi, \\
& u_{2 i}^{j}=-x_{2 i-1}^{(j)} \sin (2 i-1) \varphi+x_{2 i}^{(j)} \cos (2 i-1) \varphi,
\end{aligned}
$$

$i=1, \ldots, m ; j=0, \ldots, 2 m-1$. Then

$$
F_{\vec{y}, t}(\varphi)=\operatorname{det}\left(\begin{array}{ccccc}
u_{1}^{0} & u_{1}^{1} & \ldots & u_{1}^{2 m-1} & \widetilde{y}_{1} \\
\vdots & \vdots & & \vdots & \vdots \\
u_{2 m}^{0} & u_{2 m}^{1} & \ldots & u_{2 m}^{2 m-1} & \widetilde{y}_{2 m} \\
x_{2 m+1} & x_{2 m+1}^{\prime} & \ldots & x_{2 m+1}^{(2 m-1)} & y_{2 m+1}
\end{array}\right) \text {, }
$$


where $u_{1}^{j}=a_{j}, u_{2}^{j}=b_{j}$ and

$$
\begin{gathered}
\left(u_{2 i-1}^{0}, u_{2 i-1}^{1}, \ldots, u_{2 i-1}^{2 m-1}\right)=\left(1,0,-(2 i-1)^{2}, 0, \ldots,(-1)^{m-1}(2 i-1)^{2 m-2}, 0\right), \\
\left(u_{2 i}^{0}, u_{2 i}^{1}, \ldots, u_{2 i}^{2 m-1}\right)=\left(0,(2 i-1), 0,-(2 i-1)^{3}, \ldots, 0,(-1)^{m-1}(2 i-1)^{2 m-1}\right),
\end{gathered}
$$

for $i>1$.

Let $W^{k}$ be the determinant of the matrix obtained from $W$ by the deletion of the $k$-th column, if $m>1$, and be 1 , if $m=1$. Consider the smooth function $\Phi_{m}(x)$ which is equal to 1 , if $m=1$, and to

$$
\operatorname{det}\left(\begin{array}{ccccc}
1 & -x^{2} & x^{4} & \ldots & (-1)^{m-1} x^{2 m-2} \\
1 & -3^{2} & 3^{4} & \ldots & (-1)^{m-1} 3^{2 m-2} \\
1 & -5^{2} & 5^{4} & \ldots & (-1)^{m-1} 5^{2 m-2} \\
\vdots & \vdots & \vdots & & \vdots \\
1 & -(2 m-1)^{2} & (2 m-1)^{4} & \ldots & (-1)^{m-1}(2 m-1)^{2 m-2}
\end{array}\right),
$$

if $m>1$. Usually, we will omit the subscript $m$ and write $\Phi(x)$.

The next statement follows from the determinant decomposition theorem.

LEMMA 19.

$$
\sum_{k=0}^{m-1}(4 m+3)^{2 k} W^{k+1}=\Phi(4 m+3), \quad \sum_{k=0}^{m-1} W^{k+1}=\Phi(1) .
$$

Let

$$
P=-4(m+1) t \Phi(1) \Phi(4 m+3), \quad Q=\Phi^{2}(1)+(4 m+3) t^{2} \Phi^{2}(4 m+3) .
$$

LEMMA 20. Flattening points of the curve $\gamma_{t}$ are solutions (with the multiplicities taken into account) of the equation

$$
P \cos 2(2 m+1) \varphi+Q=0 .
$$

Proof. Flattening points of the curve $\gamma_{t}$ are solutions of the equation $F_{\vec{y}, t}(\varphi)=0$, where $\vec{y}=(0, \ldots, 0,1)$. By Lemma 18 , it is equivalent to the equation

$$
\operatorname{det}\left(\begin{array}{ll}
a_{0} & a_{1} \\
b_{0} & b_{1}
\end{array}\right)=0
$$

if $m=1$, and

$$
\operatorname{det}\left(\begin{array}{cc}
A_{0} & A_{1} \\
B_{0} & B_{1} \\
W & 0 \\
0 & W
\end{array}\right)=0,
$$

if $m>1$. Both these equations are equivalent to the equation

$$
\sum_{i, j=0}^{m-1}(-1)^{i+j} \operatorname{det}\left(\begin{array}{ll}
a_{2 i} & a_{2 j+1} \\
b_{2 i} & b_{2 j+1}
\end{array}\right) W^{i+1} W^{j+1}=0,
$$


which can be rewritten in the form

$$
\begin{aligned}
\left(\sum_{i=0}^{m-1} W^{i+1}\right)^{2}+ & (4 m+3) t^{2}\left(\sum_{i=0}^{m-1}(4 m+3)^{2 i} W^{i+1}\right)^{2} \\
& -4(m+1) t \sum_{i=0}^{m-1} W^{i+1} \sum_{i=0}^{m-1}(4 m+3)^{2 i} W^{i+1} \cos 2(2 m+1) \varphi=0
\end{aligned}
$$

by Lemma 18. Now, Lemma 20 follows from Lemma 19.

LEMMA 21. If $m>1$, then

$$
\Phi(x)=(-4)^{(m-2)(m-1) / 2} \frac{\prod_{i=2}^{m}(2 i-2) !}{(m-1) ! m !} \prod_{i=2}^{m}\left(x^{2}-(2 i-1)^{2}\right) .
$$

The proof is a direct calculation of the Vandermonde determinant which defines the function $\Phi(x)$.

Let us consider the numbers

$$
t_{0}=(-1)^{m-1} \frac{\Phi(1)}{(4 m+3) \Phi(4 m+3)}, \quad t_{1}=(-1)^{m-1} \frac{\Phi(1)}{\Phi(4 m+3)} .
$$

LEMMA 22. $0<t_{0}<t_{1}$ and

$$
t_{0}=\frac{2(2 m+1)(m-1) !((m+1) !)^{2}}{(4 m+3)(3 m+1) !} \leq \frac{1}{7} .
$$

These formulas follow from Lemma 21 (the estimate for $t_{0}$ follows from the fact that $t_{0}$ decreases when $m$ increases).

Lemma 23. The equation

$$
(-1)^{m-1} P+Q=0
$$

is quadratic with respect to $t$ and has two solutions $t_{0}$ and $t_{1}$.

Proof. The coefficient at $t^{2}$ in this equation is equal to

$$
(4 m+3) \Phi^{2}(4 m+3)
$$

and hence, is not equal to 0 by Lemma 21 . The discriminant of the equation equals

$$
4(2 m+1)^{2} \Phi^{2}(1) \Phi^{2}(4 m+3),
$$

i.e. is a perfect square.

7.2. Proof of Proposition 3. By Lemma 21, the equation from Lemma 20 is linear with respect to $\cos 2(2 m+1) \varphi$ for all $0<t<1$. Its coefficients have the following signs:

$$
(-1)^{m} P>0, \quad Q>0 .
$$

Since this equation has no solutions for $t=0$ and $P, Q$ smoothly depend on $t$, the solutions which can appear when $t$ increases must satisfy equation (4) at the moment of the appearance. Hence, this moment is the smallest positive solution of the equation $(-1)^{m-1} P+Q=0$, less than 1 . By Lemmas 22 and 23 , such a solution exists and is equal to $t_{0}$. 
It remains to remark that the expression $(-1)^{m-1} P+Q$ changes its sign when $t$ passes through $t_{0}$. If $t=t_{0}$ and $\varphi_{0}$ is a solution of equation (4), then the first derivative by $\varphi$ of the left-hand side of the equation from Lemma 20 at $\varphi_{0}$ vanishes, and the second derivative is not zero (it is equal to $\left.4(m+1)^{2}(-1)^{m-1} P\right)$. Therefore, this equation has $4 m+2$ solutions for any $t>t_{0}$ sufficiently close to $t_{0}$.

7.3. Lemmas to the proof of Proposition $5(n=2 m-1)$. Let

$$
\begin{gathered}
A_{0}^{0}=\left(\begin{array}{llll}
a_{0}^{0} & a_{2}^{0} & \ldots & a_{2 m-2}^{0}
\end{array}\right), \quad a_{2 k}^{0}=(-1)^{k}\left[1+(-1)^{m}(4 m+3)^{2 k} t\right], \\
B_{1}^{0}=\left(\begin{array}{llll}
b_{1}^{0} & b_{3}^{0} & \ldots & b_{2 m-1}^{0}
\end{array}\right), \quad b_{2 k+1}^{0}=(-1)^{k}\left[1+(-1)^{m}(4 m+3)^{2 k+1} t\right] .
\end{gathered}
$$

LEMMA 24. If $m>1$, then

$$
\begin{aligned}
\operatorname{det}\left(\begin{array}{c}
A_{0}^{0} \\
W
\end{array}\right)= & \Phi(1)\left(1-\frac{t}{t_{1}}\right), \quad \operatorname{det}\left(\begin{array}{c}
B_{1}^{0} \\
W
\end{array}\right)=\Phi(1)\left(1-\frac{t}{t_{0}}\right), \\
& \operatorname{det}\left(\begin{array}{c}
W \\
C
\end{array}\right)=(-1)^{m-1} \Phi(2 m+1) .
\end{aligned}
$$

Proof. By Lemma 19,

$$
\begin{gathered}
\operatorname{det}\left(\begin{array}{l}
A_{0}^{0} \\
W
\end{array}\right)=\Phi(1)+(-1)^{m} \Phi(4 m+3) t=\Phi(1)\left(1-\frac{t}{t_{1}}\right), \\
\operatorname{det}\left(\begin{array}{l}
B_{1}^{0} \\
W
\end{array}\right)=\Phi(1)+(-1)^{m}(4 m+3) \Phi(4 m+3) t=\Phi(1)\left(1-\frac{t}{t_{0}}\right) .
\end{gathered}
$$

The third equality is evident.

Lemma 25. Let $\varphi_{0}$ be a solution of equation (4). Then $F_{\vec{y}, t}\left(\varphi_{0}\right)$ is equal to

$$
(1-t)\left[f^{\prime}\left(\varphi_{0}\right)\left(y_{1} \sin \varphi_{0}-y_{2} \cos \varphi_{0}\right)+(1-7 t) y_{3}\right]
$$

if $m=1$, and

$$
\begin{aligned}
& \Phi(1)(2 m-1) ! !\left(1-\frac{t}{t_{1}}\right)\left\{f ^ { \prime } ( \varphi _ { 0 } ) \left[\Phi(2 m+1)\left(y_{1} \sin \varphi_{0}-y_{2} \cos \varphi_{0}\right)\right.\right. \\
& \left.\left.-\sum_{i=2}^{m} \frac{y_{2 i-1} \sin (2 i-1) \varphi_{0}-y_{2 i} \cos (2 i-1) \varphi_{0}}{2 i-1} \operatorname{det}\left(\begin{array}{cc}
B_{1}^{0} & 0 \\
W & e_{i} \\
C & 0
\end{array}\right)\right]+\Phi(1)\left(1-\frac{t}{t_{0}}\right) y_{2 m+1}\right\},
\end{aligned}
$$

if $m>1$, where $e_{i}$ is the $(m-1)$-element column whose $(i-1)$-th element is equal to 1 , and all other are equal to 0.

Proof. By Lemma 18

$$
F_{\vec{y}, t}\left(\varphi_{0}\right)=\left\{\begin{array}{ccc}
\operatorname{det}\left(\begin{array}{ccc}
a_{0}^{0} & 0 & \widetilde{y}_{1} \\
0 & b_{1}^{0} & \widetilde{y}_{2} \\
0 & f^{\prime}\left(\varphi_{0}\right) & y_{3}
\end{array}\right), & \text { if } m=1, \\
(-1)^{m-1}(2 m-1) ! ! \operatorname{det}\left(\begin{array}{ccc}
A_{0}^{0} & 0 & \widetilde{y}_{1} \\
0 & B_{1}^{0} & \widetilde{y}_{2} \\
W & 0 & Y_{+} \\
0 & W & Y_{-} \\
0 & f^{\prime}\left(\varphi_{0}\right) C & y_{2 m+1}
\end{array}\right), \quad \text { if } m>1 .
\end{array}\right.
$$


Therefore $F_{\vec{y}, t}\left(\varphi_{0}\right)=a_{0}^{0}\left[b_{1}^{0} y_{3}-f^{\prime}\left(\varphi_{0}\right) \widetilde{y}_{2}\right]$, if $m=1$, and

$F_{\vec{y}, t}\left(\varphi_{0}\right)=(2 m-1) ! ! \operatorname{det}\left(\begin{array}{c}A_{0}^{0} \\ W\end{array}\right)\left[(-1)^{m} \widetilde{y}_{2} f^{\prime}\left(\varphi_{0}\right) \operatorname{det}\left(\begin{array}{c}W \\ C\end{array}\right)+\operatorname{det}\left(\begin{array}{cc}B_{1}^{0} & 0 \\ W & Y_{-} \\ f^{\prime}\left(\varphi_{0}\right) C & y_{2 m+1}\end{array}\right)\right]$,

if $m>1$. Now, Lemma 25 follows from Lemma 24 .

7.4. Proof of Proposition $5(n=2 m-1)$. Let $\varphi_{0}$ be a flattening point of the curve $\gamma_{t_{0}}$. Then the function $F_{\vec{y}, t_{0}}\left(\varphi_{0}\right)$ of the variable $\vec{y}$ is not equal to 0 identically by Proposition 3 , Lemmas 22 and 25. Hence, an osculating hyperplane to the curve $\Gamma_{t_{0}}^{*}$ at the point $\varphi_{0}$ is unique.

Now, consider the curve $\gamma_{t}$. An osculating hyperplane to $\gamma_{t}$ at the point $\varphi_{0}$ is unique if and only if the leading $2 m-1$ columns of the matrix

$$
\left(\begin{array}{cccc}
x_{1} & x_{1}^{\prime} & \ldots & x_{1}^{(2 m-1)} \\
\vdots & \vdots & & \vdots \\
x_{2 m} & x_{2 m}^{\prime} & \ldots & x_{2 m}^{(2 m-1)}
\end{array}\right)
$$

are linearly independent. By Lemma 18, the last holds if and only if the leading $2 m-1$ columns of the matrix

$$
\Lambda=\left\{\begin{array}{cc}
\left(\begin{array}{cc}
a_{0}^{0} & 0 \\
0 & b_{1}^{0}
\end{array}\right), & \text { if } m=1, \\
\left(\begin{array}{cc}
A_{0}^{0} & 0 \\
0 & B_{1}^{0} \\
W & 0 \\
0 & W
\end{array}\right), & \text { if } m>1,
\end{array}\right.
$$

are linearly independent. But the determinant of the matrix obtained from $\Lambda$ by the deletion of the second line and the last column is equal to

$$
\chi= \begin{cases}a_{0}^{0}, & \text { if } m=1 \\
\operatorname{det}\left(\begin{array}{c}
A_{0}^{0} \\
W
\end{array}\right) \operatorname{det} W^{m}, & \text { if } m>1\end{cases}
$$

where $a_{0}^{0}=1-t$ (if $m=1$ ) and $\operatorname{det} W^{m} \neq 0$ (the Vandermonde determinant). According to Lemmas 22 and $24, \chi \neq 0$ for $t=t_{0}$. Hence, an osculating hyperplane to the curve $\gamma_{t_{0}}$ at the point $\varphi_{0}$ is unique.

7.5. Lemmas to the proof of Proposition 4. Let

$$
\begin{array}{r}
\Psi(x)=\operatorname{det}\left(\begin{array}{ccccc}
1 & -x^{2} & x^{4} & \ldots & (-1)^{m} x^{2 m} \\
1 & -1^{2} & 1^{4} & \ldots & (-1)^{m} 1^{2 m} \\
1 & -3^{2} & 3^{4} & \ldots & (-1)^{m} 3^{2 m} \\
\vdots & \vdots & \vdots & & \vdots \\
1 & -(2 m-1)^{2} & (2 m-1)^{4} & \ldots & (-1)^{m}(2 m-1)^{2 m}
\end{array}\right), \\
\widetilde{P}=-t\left[(4 m+3) \Phi_{m+1}(1) \Phi(4 m+3)+\Phi(1) \Phi_{m+1}(4 m+3)\right. \\
\left.+(-1)^{m-1}(2 m+1) \Phi(2 m+1) \Psi(4 m+3)\right], \\
\widetilde{Q}=\Phi_{m+1}(1) \Phi(1)-t(2 m+1) \Phi(2 m+1) \Psi(4 m+3) \\
+t^{2}(4 m+3) \Phi_{m+1}(4 m+3) \Phi(4 m+3) .
\end{array}
$$


LEMMA 26. Flattening points of the curve $\Gamma_{t}$ are solutions (with the multiplicities taken into account) of the equation

$$
f(\varphi)[\widetilde{P} \cos 2(2 m+1) \varphi+\widetilde{Q}]=0 .
$$

Proof. These points are solutions of the equation $F_{\vec{y}, t}(\varphi)=0$ where

$$
\vec{y}=\left(x_{1}^{(2 m)}, \ldots, x_{2 m+1}^{(2 m)}\right) .
$$

By Lemma 18, it is equivalent to the equation

$$
\operatorname{det}\left(\begin{array}{ccc}
a_{0} & a_{1} & a_{2} \\
b_{0} & b_{1} & b_{2} \\
f(\varphi) & f^{\prime}(\varphi) & y_{3}
\end{array}\right)=0
$$

if $m=1$, and

if $m>1$, where

$$
\operatorname{det}\left(\begin{array}{ccc}
A_{0} & A_{1} & a_{2 m} \\
B_{0} & B_{1} & b_{2 m} \\
W & 0 & Y_{+} \\
0 & W & 0 \\
f(\varphi) C & f^{\prime}(\varphi) C & y_{2 m+1}
\end{array}\right)=0
$$

$$
Y_{+}=(-1)^{m}\left(\begin{array}{c}
3^{2 m} \\
\vdots \\
(2 m-1)^{2 m}
\end{array}\right), \quad y_{2 m+1}=(-1)^{m}(2 m+1)^{2 m} f(\varphi) .
$$

The left-hand sides of the indicated equations are equal to

$$
\begin{gathered}
f(\varphi) \operatorname{det}\left(\begin{array}{ccc}
a_{0} & a_{1} & a_{2} \\
b_{0} & b_{1} & b_{2} \\
1 & 0 & -9
\end{array}\right)+f^{\prime}(\varphi) \operatorname{det}\left(\begin{array}{ccc}
a_{0} & a_{1} & a_{2} \\
b_{0} & b_{1} & b_{2} \\
0 & 1 & 0
\end{array}\right) \\
\quad=f(\varphi)\left[\operatorname{det}\left(\begin{array}{c}
\widetilde{A}_{0} \\
W_{2}
\end{array}\right) b_{1}-\operatorname{det}\left(\begin{array}{c}
\widetilde{B}_{0} \\
W_{2}
\end{array}\right) a_{1}\right]-f^{\prime}(\varphi) \operatorname{det}\left(\begin{array}{cc}
a_{0} & a_{2} \\
b_{0} & b_{2}
\end{array}\right)
\end{gathered}
$$

and

$$
\begin{array}{r}
f(\varphi) \operatorname{det}\left(\begin{array}{ccc}
A_{0} & A_{1} & a_{2 m} \\
B_{0} & B_{1} & b_{2 m} \\
W & 0 & Y_{+} \\
0 & W & 0 \\
C & 0 & (-1)^{m}(2 m+1)^{2 m}
\end{array}\right)+f^{\prime}(\varphi) \operatorname{det}\left(\begin{array}{ccc}
A_{0} & A_{1} & a_{2 m} \\
B_{0} & B_{1} & b_{2 m} \\
W & 0 & Y_{+} \\
0 & W & 0 \\
0 & C & 0
\end{array}\right) \\
=(-1)^{m-1} f(\varphi)\left[\operatorname{det}\left(\begin{array}{c}
\widetilde{A}_{0} \\
W_{m+1}
\end{array}\right) \operatorname{det}\left(\begin{array}{c}
B_{1} \\
W
\end{array}\right)-\operatorname{det}\left(\begin{array}{c}
\widetilde{B}_{0} \\
W_{m+1}
\end{array}\right) \operatorname{det}\left(\begin{array}{c}
A_{1} \\
W
\end{array}\right)\right] \\
+(-1)^{m} f^{\prime}(\varphi) \operatorname{det}\left(\begin{array}{cc}
A_{0} & a_{2 m} \\
B_{0} & b_{2 m} \\
W & Y_{+}
\end{array}\right) \operatorname{det}\left(\begin{array}{c}
W \\
C
\end{array}\right),
\end{array}
$$

respectively, where

$$
\widetilde{A}_{0}=\left(\begin{array}{llll}
a_{0} & a_{2} & \ldots & a_{2 m}
\end{array}\right), \quad \widetilde{B}_{0}=\left(\begin{array}{llll}
b_{0} & b_{2} & \ldots & b_{2 m}
\end{array}\right) .
$$


By Lemma 19 and the definition of the function $\Psi$, we have:

$$
\begin{aligned}
\operatorname{det}\left(\begin{array}{c}
\widetilde{A}_{0} \\
W_{m+1}
\end{array}\right) & =\Phi_{m+1}(1)-t \Phi_{m+1}(4 m+3) \cos 2(2 m+1) \varphi, \\
\operatorname{det}\left(\begin{array}{c}
\widetilde{B}_{0} \\
W_{m+1}
\end{array}\right) & =-t \Phi_{m+1}(4 m+3) \sin 2(2 m+1) \varphi, \\
\operatorname{det}\left(\begin{array}{c}
A_{1} \\
W
\end{array}\right) & =t(4 m+3) \Phi(4 m+3) \sin 2(2 m+1) \varphi, \\
\operatorname{det}\left(\begin{array}{c}
B_{1} \\
W
\end{array}\right) & =\Phi(1)-t(4 m+3) \Phi(4 m+3) \cos 2(2 m+1) \varphi, \\
\operatorname{det}\left(\begin{array}{cc}
A_{0} & a_{2 m} \\
B_{0} & b_{2 m} \\
W & Y_{+}
\end{array}\right) & =t \Psi(4 m+3) \sin 2(2 m+1) \varphi .
\end{aligned}
$$

These formulas and Lemma 24 imply the above equation for flattening points of the curve $\Gamma_{t}$.

LEMMA 27.

$$
\Psi(x)=(-4)^{m(m-1) / 2} \prod_{i=1}^{m}(2 i-2) ! \prod_{i=1}^{m}\left(x^{2}-(2 i-1)^{2}\right) .
$$

The proof is a direct calculation of the Vandermonde determinant which defines the function $\Psi(x)$.

Lemma 28. $\widetilde{P}<0$ for any $t>0$.

Proof. By Lemmas 21 and 27,

$$
\begin{gathered}
(4 m+3) \Phi_{m+1}(1) \Phi(4 m+3)+(-1)^{m-1}(2 m+1) \Phi(2 m+1) \Psi(4 m+3) \\
=\frac{4^{m^{2}}(2 m) !(3 m+1) !}{2 m !((m+1) !)^{2}(2 m+1)}\left(\prod_{i=1}^{m}(2 i-2) !\right)^{2}\left(4 m^{2}+5 m+2\right)>0, \\
\Phi(1) \Phi_{m+1}(4 m+3)>0 .
\end{gathered}
$$

Therefore, $\widetilde{P}<0$, if $t$ is positive.

The following statement is evident.

Lemma 29. $(-1)^{m} \widetilde{Q}>0$ for any $t$ sufficiently close to 0 .

Let us consider the numbers

$$
T_{0}=(-1)^{m} \frac{\Phi_{m+1}(1)}{\Phi_{m+1}(4 m+3)}, \quad T_{1}=(-1)^{m} \frac{\Phi(1)}{(4 m+3) \Phi(4 m+3)} .
$$

LEMMA $30 . T_{1}<0, T_{0}>t_{0}$ and

$$
T_{0}=\frac{2(2 m+1)(m+1)^{2}(m !)^{3}}{(3 m+2) !} \leq \frac{1}{5} .
$$

These formulas follow from Lemmas 21 and 22 . 
LEMMA 31. The equation

$$
(-1)^{m} \widetilde{P}+\widetilde{Q}=0
$$

is quadratic with respect to $t$ and has two solutions $T_{0}$ and $T_{1}$.

Proof. The coefficient at $t^{2}$ in this equation is equal to

$$
(4 m+3) \Phi_{m+1}(4 m+3) \Phi(4 m+3)
$$

and hence, is not equal to 0 by Lemma 21 . The discriminant of the equation is

$$
\left[(4 m+3) \Phi_{m+1}(1) \Phi(4 m+3)-\Phi(1) \Phi_{m+1}(4 m+3)\right]^{2},
$$

i.e. is a perfect square.

7.6. Proof of Proposition 4. By Lemma 26, flattening points of the curve $\Gamma_{t}$ are defined by the equations $f(\varphi)=0$ and

$$
\widetilde{P} \cos 2(2 m+1) \varphi+\widetilde{Q}=0 .
$$

The first one has $2 m+1$ solutions $\cos 2(2 m+1) \varphi=(-1)^{m-1}$. The second one has no solutions if $t=0$ and is quadratic with respect to $\cos 2(2 m+1) \varphi$ for every $t \neq 0$.

Since $\widetilde{P}, \widetilde{Q}$ smoothly depend on $t$ and satisfy the inequalities from Lemmas 28, 29, the solutions which can appear from the second equation when $t$ increases from 0 to 1 must satisfy the equation $\cos 2(2 m+1) \varphi=(-1)^{m}$ at the moment of the appearance. Hence, this moment is the smallest positive solution of the equation $(-1)^{m} \widetilde{P}+\widetilde{Q}=0$, less than 1. By Lemmas 30 and 31, such a solution exists and is equal to $T_{0}$ and greater than $t_{0}$.

It remains to remark that for any $t \in\left[0, T_{0}\right)$ and any solution $\varphi_{0}$ of equation (4), the first derivative by $\varphi$ of the left-hand side of the equation from Lemma 26 at $\varphi_{0}$ is not equal to 0 .

7.7. Lemmas to the proofs of Propositions $6-8(n=2 m-1)$. The osculating hyperplane to the curve $\Gamma_{t}$ at a point $\varphi$ is defined by the equation

$$
F_{\vec{y}, t}(\varphi)=0,
$$

where $\vec{y}=\left(y_{1}, \ldots, y_{2 m+1}\right)$ and $\left(y_{1}: \ldots: y_{2 m+1}\right) \in \mathbb{R} P^{2 m}$.

LEMma 32. If $t$ is sufficiently close to $t_{0}$, then the determinant

$$
\operatorname{det}\left(\begin{array}{cc}
B_{1}^{0} & 0 \\
W & e_{i} \\
C & 0
\end{array}\right)
$$

from Lemma 25 is not equal to 0 for any $i=2, \ldots, m$.

Proof. Let us consider the more general determinant

$$
\operatorname{det}\left(\begin{array}{cc}
B_{1}^{0} & 0 \\
W & \delta_{i}^{*} \\
C & \delta_{i}^{m+1}
\end{array}\right), \quad i \in\{2, \ldots, m+1\},
$$


where the $i$-th element of the last column equals 1, and all other equal 0. By Vandermonde formula, this determinant is equal to

$$
\begin{aligned}
(-1)^{i} \prod_{\substack{2 \leq k<l \leq m+1 \\
k \neq i, l \neq i}}\left((2 k-1)^{2}\right. & \left.-(2 l-1)^{2}\right)\left[\frac{1}{(2 i-1)^{2}-1} \prod_{j=2}^{m+1}\left((2 j-1)^{2}-1\right)\right. \\
& \left.-\frac{t(4 m+3)}{(4 m+3)^{2}-(2 i-1)^{2}} \prod_{j=2}^{m+1}\left((4 m+3)^{2}-(2 j-1)^{2}\right)\right] .
\end{aligned}
$$

This expression vanishes for $t=\tau_{i}(m)$ where

$$
\tau_{i}(m)=\frac{(4 m+3)^{2}-(2 i-1)^{2}}{(2 i-1)^{2}-1} \cdot \frac{\prod_{j=2}^{m+1}\left((2 j-1)^{2}-1\right)}{(4 m+3) \prod_{j=2}^{m+1}\left((4 m+3)^{2}-(2 j-1)^{2}\right)} .
$$

It is evident that $\tau_{2}(m)>\ldots>\tau_{m}(m)>\tau_{m+1}(m)$ since the function $\frac{\lambda-x}{x-1}$ increases on the interval $x \in(1,+\infty)$ for any $\lambda>1$. It remains to remark that $\tau_{m+1}(m)=t_{0}$ by Lemma 24.

Remark 13. The moments $\tau_{i}(m), i=2, \ldots, m$, as well as the moments $t_{0}, T_{0}$, define some bifurcations in the family $\Gamma_{t}$. In particular, the beginning of the sequence $\tau_{m}(m)$ for $m>1$ is:

$$
\frac{1}{44}, \quad \frac{1}{1485}, \quad \frac{1}{40964}, \quad \frac{1}{1067430}, \quad \frac{1}{27264600}, \quad \ldots .
$$

It is easy to see that $\tau_{m}(m)<T_{0}=T_{0}(m)$ already for $m=3$.

7.8. Proof of Proposition $6(n=2 m-1)$. Let $\varphi_{1}, \ldots, \varphi_{2 m}$ be mutually different solutions of equation (4). Then $\Theta$ is the unique solution of the linear equations system

$$
F_{\vec{y}, t_{0}}\left(\varphi_{i}\right)=0, \quad i=1, \ldots, 2 m \text {. }
$$

Indeed, the point $\Theta$ satisfies this system by Lemma 25. The rank of the system is equal to the rank of the matrix formed by the coordinates of the vectors

$$
\left(\cos \varphi_{i}, \sin \varphi_{i}, \cos 3 \varphi_{i}, \sin 3 \varphi_{i}, \ldots, \cos (2 m-1) \varphi_{i}, \sin (2 m-1) \varphi_{i}\right), \quad i=1, \ldots, 2 m,
$$

(this follows from Lemmas 21, 22 and 32). But the determinant of this matrix is not 0 (even for any mutually different points $\varphi_{1}, \ldots, \varphi_{2 m}$ on the circle $\{\varphi \bmod \pi\}$ ) since the curve $\gamma_{0}$ is convex.

7.9. Proof of Proposition $7(n=2 m-1)$. Let us consider the solutions

$$
\varphi_{i}=\frac{1+(-1)^{m}}{4(2 m+1)} \pi+\frac{\pi}{2 m+1} i, \quad i=0,1, \ldots, 2 m,
$$

of equation (4). The intersection of the osculating hyperplanes to the curve $\Gamma_{t}$ at these points is defined by the linear equations system

$$
F_{\vec{y}, t}\left(\varphi_{i}\right)=0, \quad i=0,1, \ldots, 2 m
$$

If $t$ is sufficiently close to $t_{0}$ and $t \neq t_{0}$, then by Lemmas $21,22,25$ and 32 , the rank of this system is equal to the rank of the matrix formed by the coordinates of the vectors

$$
v_{i}=\left(\cos \varphi_{i}, \sin \varphi_{i}, \cos 3 \varphi_{i}, \sin 3 \varphi_{i}, \ldots, \cos (2 m-1) \varphi_{i}, \sin (2 m-1) \varphi_{i}, f^{\prime}\left(\varphi_{i}\right)\right),
$$


$i=0,1, \ldots, 2 m$.

Let us suppose that these vectors lie in a hyperplane in $\mathbb{R}^{2 m+1}$. Then this hyperplane contains the vector $(1,0, \ldots, 0)$ since

Hence, the vectors

$$
\frac{(-1)^{m-1}}{2 m+1} \sum_{i=0}^{2 m}(-1)^{i} v_{i}=(0, \ldots, 0,1) .
$$

$$
\widetilde{v}_{i}=\left(\cos \varphi_{i}, \sin \varphi_{i}, \cos 3 \varphi_{i}, \sin 3 \varphi_{i}, \ldots, \cos (2 m-1) \varphi_{i}, \sin (2 m-1) \varphi_{i}\right),
$$

$i=0,1, \ldots, 2 m$, lie in a hyperplane in $\mathbb{R}^{2 m}$. But this contradicts the convexity of the curve $\gamma_{0}$.

7.10. Proof of Proposition $8(n=2 m-1)$. Let $\vec{y}=(0, \ldots, 0,1) \in \mathbb{R}^{2 m+1}$ and $\varphi$ be a solution of equation (4). Then

$$
F_{\vec{y}, t}(\varphi)=\Phi^{2}(1)(2 m-1) ! !\left(1-\frac{t}{t_{1}}\right)\left(1-\frac{t}{t_{0}}\right)
$$

by Lemma 25. Now, the statement follows from Lemmas 21 and 22 .

\section{References}

[An] S. Anisov, Projective convex curves, in: Arnold-Gelfand Mathematical Seminars. Geometry and Singularity Theory, V. I. Arnol'd, I. M. Gel'fand, V. S. Retakh and M. Smirnov (eds.), Birkhäuser, Boston, 1997, 93-99.

[AGV] V. I. Arnol'd, S. M. Guse ̌̆n-Zade, A. N. Varchenko, Singularities of Differentiable Maps I, Monogr. Math. 82, Birkhäuser, Boston, 1985.

[Ar] V. I. Arnol'd, On the number of flattening points of space curves, in: Sinal's Moscow Seminar on Dynamical Systems, Amer. Math. Soc. Transl. Ser. 2, 171, Amer. Math. Soc., Providence, 1996, 11-22.

[B] M. Barner, Über die Mindestanzahl stationärer Schmiegebenen bei geschlossenen strengkonvexen Raumkurven, Abh. Math. Sem. Univ. Hamburg 20 (1956), 196-215.

[H] A. Hurwitz, Über die Fourierschen Konstanten integrierbarer Funktionen, Math. Ann. 57 (1903), 425-446.

[Sch] I. J. Schoenberg, An isoperimetric inequality for closed curves convex in even-dimensional Euclidean spaces, Acta Math. 91 (1954), 143-164. 